\title{
The fully differential decay rate of a Higgs boson to bottom-quarks at NNLO in QCD
}

\section{Journal Article}

\section{Author(s):}

Anastasiou, Charalampos; Herzog, Franz; Lazopoulos, Achilleas

Publication date:

2012-03

Permanent link:

https://doi.org/10.3929/ethz-b-000048659

\section{Rights / license:}

Creative Commons Attribution 4.0 International

\section{Originally published in:}

Journal of High Energy Physics 2012(3), https://doi.org/10.1007/JHEP03(2012)035 


\title{
The fully differential decay rate of a Higgs boson to bottom-quarks at NNLO in QCD
}

\author{
Charalampos Anastasiou, Franz Herzog and Achilleas Lazopoulos \\ Institute for Theoretical Physics, ETH Zurich, \\ 8093 Zurich, Switzerland \\ E-mail: babis@phys.ethz.ch, fherzog@itp.phys.ethz.ch, \\ lazopoli@itp.phys.ethz.ch
}

ABSTRACT: The decay of a light Higgs boson to bottom quarks is dominant and can be exploited for the discovery of the Higgs particle and the measurement of its properties at the LHC and future collider experiments. We perform a first computation of the fully differential decay at next-next-to-leading order in perturbative QCD. We employ a novel method of non-linear mappings for the treatment of singularities in the radiative processes which contribute to the decay width. This constitutes the first physical application of the method.

Keywords: NLO Computations, QCD, Higgs Physics, Jets

ARXIV EPRINT: 1110.2368 


\section{Contents}

1 Introduction 1

2 Notation 2

3 Matrix elements $\quad 4$

3.1 Decay to two partons 4

$\begin{array}{lll}3.2 & \text { Decay to three partons } & 6\end{array}$

$\begin{array}{lll}3.3 & \text { Decay to four partons } & 7\end{array}$

$\begin{array}{llr}4 & \text { Integration over phase space } & 8\end{array}$

$\begin{array}{lll}5 & \text { Real-virtual NNLO contribution } & 9\end{array}$

$\begin{array}{lll}6 & \text { Integration of double-real contributions } & 11\end{array}$

$\begin{array}{lll}7 & \text { Numerical results } & 13\end{array}$

8 Conclusions $\quad 15$

$\begin{array}{ll}\text { A Double-real matrix elements } & 15\end{array}$

\section{Introduction}

Direct searches and electroweak precision tests suggest that a Standard Model Higgs boson is light, and that it should decay predominantly into a bottom quark $(b \bar{b})$ pair. Inclusive searches at the LHC of a Higgs resonance in the bottom-pair invariant mass distribution are unfortunately hampered by a very large QCD background. Direct searches are focusing on other decay channels, such as $H \rightarrow \gamma \gamma$, with a much smaller branching ratio. In view of this, perturbative corrections to the $H \rightarrow b \bar{b}$ decay are most interesting for the inclusive rate, due to its contribution to the total decay width and the branching ratios of other decays. A remarkable effort has been made for a precise calculation of $\Gamma_{H \rightarrow b \bar{b}}$ and now QCD corrections are known to $\mathcal{O}\left(\alpha_{s}^{4}\right)[1-9]$.

Recently, confidence has grown that the $H \rightarrow b \bar{b}$ decay can be observed at the LHC directly in events where the Higgs boson is produced in association with a massive vector boson $(W, Z)[10]$ or a $t \bar{t}$ pair [11]. Backgrounds from $t \bar{t}, V+j j$ and multi-jet production are challenging. However, the excellent $b$-jet tagging of the ATLAS and CMS detectors as well as sophisticated selections of jet events described in [10,11], render these channels hopeful, as explicitly demonstrated by the ATLAS collaboration in a full detector simulation analysis for the $Z H$ case [12]. For this channel, further suppression of the background can be obtained with the sub-jet algorithms of [13]. 
These search strategies rely on a selection of phase space corners which are rich in potential Higgs events. An accurate modeling of QCD radiation is necessary in order to assess the efficiency of these selections. This motivates the computation of the fully differential $H \rightarrow b \bar{b}$ decay rate at next-to-next-to-leading-order (NNLO) in perturbative QCD.

Next-to-leading-order (NLO) computations [14-26] are currently performed with very well automated methods [27-35]. Obtaining fully differential cross-sections and decay rates at one order higher in the perturbative expansion requires the solution of new challenging problems. In the last decade rapid progress [36-60] with important results [61-73] has been achieved. However, further developments of methods and new ideas are necessary for efficient cancelations of infrared singularities and evaluations of novel two-loop amplitudes in more complicated LHC processes.

In ref. [74] we introduced a new method to extract the singularities of multi-dimensional loop and phase space integrals which emerge at NNLO. The method employs non-linear transformations for the factorization of overlapping singularities which in turn allow for an efficient numerical evaluation. In this article, we demonstrate the method in a realistic physical example. We compute for the first time the fully differential decay rate for $H \rightarrow b \bar{b}$ at NNLO.

The paper is organized as follows. In section 2 we fix our notation and set up the calculation. In section 3 we present the matrix elements for the processes which contribute to the decay width through NNLO. In section 4 we describe the parameterization of the phase space for the final state partons. In section 5 and section 6 we explain how our method leads to a simple factorizable form for the singularities in the integrals which emerge at NNLO. In section 7 we present the inclusive width and examples of differential observables with our numerical code.

\section{Notation}

We compute the partial width $\Gamma_{H \rightarrow b \bar{b} X}[\mathcal{J}]$ through NNLO in perturbative QCD for any infrared safe observable $\mathcal{J}$, such as an appropriate jet-algorithm, in the decay of a Higgs boson to a pair of bottom-quarks,

$$
H \rightarrow b+\bar{b}+X
$$

We consider that the Higgs boson couples to a bottom-quark pair with an unrenormalized Yukawa coupling $y_{b}^{B}$. Due to the largely different mass of the Higgs boson, $m_{H}$, and the small bottom-quark, $m_{b}$, we treat the latter as independent of the Yukawa coupling and set it to zero, $m_{b}=0$, in matrix elements and phase space integrals. In addition, we neglect top-quarks both in virtual corrections and in the renormalization procedure and consider $n_{f}=5$ light quark flavors. For the renormalization of ultraviolet (UV) divergences we work in the $\overline{\mathrm{MS}}$-scheme where the bare couplings, $y_{b}^{B}$, and $g_{s}^{B}=\sqrt{4 \pi \alpha_{s}^{B}}$, are related to 
their renormalized counterparts through the following relations:

$$
\begin{aligned}
y_{b}^{B}= & y_{b}\left[1-\frac{\alpha_{s}}{4 \pi} \frac{3 C_{F}}{\epsilon}+\left(\frac{\alpha_{s}}{4 \pi}\right)^{2}\left[-\frac{1}{\epsilon^{2}}\left(2 T_{F} n_{f}-\frac{9}{2} C_{F}-\frac{11}{2} C_{A}\right)\right.\right. \\
& \left.\left.+\frac{1}{\epsilon}\left(\frac{5 T_{F}}{3} n_{f}-\frac{3 C_{F}}{4}-\frac{97}{12} C_{A}\right)\right] C_{F}+\mathcal{O}\left(\alpha_{s}^{3}\right)\right] \\
\alpha_{s}^{B}= & \alpha_{s}\left(\frac{\mu^{2} e^{\gamma_{E}}}{4 \pi}\right)^{\epsilon}\left[1-\frac{\alpha_{s}}{4 \pi} \frac{1}{\epsilon}\left[\frac{11 C_{A}}{3}-\frac{4 T_{F} n_{f}}{3}\right]+\mathcal{O}\left(\alpha_{s}^{2}\right)\right] .
\end{aligned}
$$

The renormalized strong coupling and Higgs Yukawa coupling depend implicitly on the renormalization scale $\mu$,

$$
\alpha_{s} \equiv \alpha_{s}(\mu) \quad y_{b} \equiv y_{b}(\mu) .
$$

The SU(N) Casimirs are given by

$$
C_{A}=N, \quad C_{F}=\frac{N^{2}-1}{2 N}, \quad T_{F}=\frac{1}{2},
$$

where $N=3$ is the number of quark colors.

Through NNLO, the partial decay width receives contributions from the following partonic processes:

- $H\left(p_{H}\right) \rightarrow b\left(p_{1}\right) \bar{b}\left(p_{2}\right)$ at two loops,

- $H\left(p_{H}\right) \rightarrow b\left(p_{1}\right) \bar{b}\left(p_{2}\right) g\left(p_{3}\right)$ at one loop and

- $H\left(p_{H}\right) \rightarrow b\left(p_{1}\right) \bar{b}\left(p_{2}\right) g\left(p_{3}\right) g\left(p_{4}\right), b\left(p_{1}\right) \bar{b}\left(p_{2}\right) b\left(p_{3}\right) \bar{b}\left(p_{4}\right), b\left(p_{1}\right) \bar{b}\left(p_{2}\right) q\left(p_{3}\right) \bar{q}\left(p_{4}\right)$ with $q \neq b$ at tree level,

where in parenthesis we indicate the four-momenta of the corresponding particles. The width is the sum

$$
\begin{aligned}
\Gamma_{H \rightarrow b \bar{b} X}[\mathcal{J}]= & \Gamma_{H \rightarrow b \bar{b}}[\mathcal{J}]+\Gamma_{H \rightarrow b \bar{b} g}[\mathcal{J}] \\
& +\Gamma_{H \rightarrow b \bar{b} g g}[\mathcal{J}]+\left(n_{f}-1\right) \cdot \Gamma_{H \rightarrow b \bar{b} q q}[\mathcal{J}]+\Gamma_{H \rightarrow b \bar{b} b \bar{b}}[\mathcal{J}]+\mathcal{O}\left(\alpha_{s}^{3}\right),
\end{aligned}
$$

where

$$
\begin{aligned}
\Gamma_{H \rightarrow b \bar{b}}[\mathcal{J}] & =\frac{1}{m_{H}} \int d \Phi_{2} \sum_{\text {spin }}\left|\mathcal{M}_{H \rightarrow b\left(p_{1}\right)+\bar{b}\left(p_{2}\right)}\right|^{2} \mathcal{J}\left(p_{1}, p_{2}\right), \\
\Gamma_{H \rightarrow b \bar{b} g}[\mathcal{J}] & =\frac{1}{m_{H}} \int d \Phi_{3} \sum_{\text {spin }}\left|\mathcal{M}_{H \rightarrow b\left(p_{1}\right)+\bar{b}\left(p_{2}\right)+g\left(p_{3}\right)}\right|^{2} \mathcal{J}\left(p_{1}, p_{2}, p_{3}\right), \\
\Gamma_{H \rightarrow b \bar{b} g g}[\mathcal{J}] & =\frac{1}{m_{H}} \int d \Phi_{4} \sum_{\text {spin }}\left|\mathcal{M}_{H \rightarrow b\left(p_{1}\right)+\bar{b}\left(p_{2}\right)+g\left(p_{3}\right)+g\left(p_{4}\right)}\right|^{2} \cdot \frac{1}{2 !} \cdot \mathcal{J}\left(p_{1}, p_{2}, p_{3}, p_{4}\right), \\
\Gamma_{H \rightarrow b \bar{b} b \bar{b}}[\mathcal{J}] & =\frac{1}{m_{H}} \int d \Phi_{4} \sum_{\text {spin }}\left|\mathcal{M}_{H \rightarrow b\left(p_{1}\right)+\bar{b}\left(p_{2}\right)+b\left(p_{3}\right)+\bar{b}\left(p_{4}\right)}\right|^{2} \cdot \frac{1}{(2 !)^{2}} \cdot \mathcal{J}\left(p_{1}, p_{2}, p_{3}, p_{4}\right), \\
\Gamma_{H \rightarrow b \bar{b} q \bar{q}}[\mathcal{J}] & =\frac{1}{m_{H}} \int d \Phi_{4} \sum_{\operatorname{spin}}\left|\mathcal{M}_{H \rightarrow b\left(p_{1}\right)+\bar{b}\left(p_{2}\right)+q\left(p_{3}\right)+\bar{q}\left(p_{4}\right)}\right|^{2} \mathcal{J}\left(p_{1}, p_{2}, p_{3}, p_{4}\right) .
\end{aligned}
$$


In the above, we integrate the appropriate matrix elements computed in conventional dimensional regularization (CDR) over the phase space in $D=4-2 \epsilon$ dimensions of the final-state partons and the function $\mathcal{J}$ defining an infrared safe observable. The phase space measure reads

$$
d \Phi_{n} \equiv(2 \pi)^{D} \delta^{(D)}\left(p_{H}-\sum_{i=1}^{n} p_{i}\right) \prod_{i=1}^{n} \frac{d^{D} p_{i}}{(2 \pi)^{D-1}} \delta^{(+)}\left(p_{i}^{2}\right) .
$$

Lorentz invariants are defined as

$$
m_{H}^{2}=p_{H}^{2}, \quad s_{i j}=2 p_{i} \cdot p_{j}, \quad s_{i j k}=s_{i j}+s_{i k}+s_{j k} .
$$

Regarding the analytic continuations of complex functions presented in this paper the positive "epsilon prescription" must be used, since all occurring mass scales are external,i.e.

$$
m_{H}^{2} \rightarrow m_{H}^{2}+i \varepsilon, \quad s_{i j} \rightarrow s_{i j}+i \varepsilon .
$$

where $\varepsilon$ is a small positive parameter.

\section{Matrix elements}

In this section we present the square of the scattering amplitudes needed for the computation of the $H \rightarrow b \bar{b}$ decay width through $\mathcal{O}\left(\alpha_{s}(\mu)^{2}\right)$. These can be easily derived with modern methods for computing Feynman diagrams and have been ingredients of many prior calculations. For example, the same matrix elements enter the calculation of the NNLO total cross-section $b \bar{b} \rightarrow H$ [75]. We have made an independent computation and present them here for completeness. For the generation of the matrix elements we used QGRAF [76]. For further symbolic manipulations, such as color and Dirac algebra, we used FORM [77] and MAPLE [78]. We reduced one and two-loop amplitudes to master integrals using the method of integration by parts [79, 80] and the Laporta algorithm [81] with AIR [82].

\subsection{Decay to two partons}

For the process

$$
H\left(p_{H}\right) \rightarrow b\left(p_{1}\right)+\bar{b}\left(p_{2}\right)
$$

we require up to 2-loop corrections:

$$
\left|\mathcal{M}_{H \rightarrow b \bar{b}}\right|^{2}=2 y_{b}(\mu)^{2} m_{H}^{2} N\left[1+\frac{\alpha_{s}(\mu)}{\pi} \mathcal{A}_{H \rightarrow b \bar{b}}^{(1)}+\left(\frac{\alpha_{s}(\mu)}{\pi}\right)^{2} \mathcal{A}_{H \rightarrow b \bar{b}}^{(2)}+\mathcal{O}\left(\alpha_{s}(\mu)^{3}\right)\right],
$$

where (let $\Re(z)$ shall denote the real part of $z$ )

$$
\mathcal{A}_{H \rightarrow b \bar{b}}^{(1)}=C_{F}\left[\Re\left(\left(\frac{\mu^{2}}{-m_{H}^{2}}\right)^{\epsilon}\right) f_{\epsilon}+\frac{3}{2 \epsilon}\right]
$$


with

$$
f_{\epsilon}=(1-\epsilon)^{2} \frac{\Gamma(1+\epsilon) \Gamma(-\epsilon)^{2}}{\Gamma(2-2 \epsilon)} e^{\gamma \epsilon} .
$$

The $\mathcal{O}\left(\alpha_{s}(\mu)^{2}\right)$ contribution can be split into two pieces

$$
\mathcal{A}_{H \rightarrow b \bar{b}}^{(2)}=\mathcal{A}_{H \rightarrow b \bar{b}}^{V V}+\mathcal{A}_{H \rightarrow b \bar{b}}^{V^{2}}
$$

corresponding to the one-loop squared amplitude

$$
\mathcal{A}_{H \rightarrow b \bar{b}}^{V^{2}}=C_{F}^{2}\left[\left|\frac{\mu^{2}}{-m_{H}^{2}}\right|^{2 \epsilon} f_{\epsilon}^{2}+\frac{3}{\epsilon} \Re\left(\left(\frac{\mu^{2}}{-m_{H}^{2}}\right)^{\epsilon}\right) f_{\epsilon}+\frac{9}{4 \epsilon^{2}}\right] .
$$

and the two-loop amplitude interfered with the tree amplitude

$$
\mathcal{A}_{H \rightarrow b \bar{b}}^{V V}=\left[\frac{\mathcal{A}_{-4}^{V V}}{\epsilon^{4}}+\frac{\mathcal{A}_{-3}^{V V}}{\epsilon^{3}}+\frac{\mathcal{A}_{2}^{V V}}{\epsilon^{2}}+\frac{\mathcal{A}_{-1}^{V V}}{\epsilon}+\mathcal{A}_{0}^{V V}\right]
$$

where

$$
\begin{aligned}
\mathcal{A}_{0}^{V V}= & {\left[1 / 6 l_{H}{ }^{4}+\frac{13}{72} l_{H}{ }^{3}+\left(-6 \zeta(2)-\frac{31}{36}\right) l_{H}{ }^{2}+\left(-\frac{55}{108}-\frac{61}{24} \zeta(2)-7 / 6 \zeta(3)\right) l_{H}\right.} \\
& \left.+\frac{455}{162}+\frac{377}{72} \zeta(2)+\frac{263}{16} \zeta(4)-\frac{47}{36} \zeta(3)\right] C_{F}{ }^{2} \\
& +\left[-1 / 36 n_{f} l_{H}{ }^{3}+\frac{5}{36} n_{f} l_{H}^{2}+1 / 27(9 \zeta(2)-7) n_{f} l_{H}\right. \\
& \left.-\frac{1}{648}(495 \zeta(2)-18 \zeta(3)-200) n_{f}\right] C_{F} \\
& +\left[\frac{11}{72} l_{H}^{3}+\left(1 / 4 \zeta(2)-\frac{67}{72}\right) l_{H}^{2}+\left(-\frac{13}{4} \zeta(3)-\frac{11}{6} \zeta(2)+\frac{121}{108}\right) l_{H}\right. \\
& \left.+\frac{701}{144} \zeta(2)-\zeta(4)-\frac{467}{648}+\frac{151}{72} \zeta(3)\right] C_{F} N^{-1} \\
\mathcal{A}_{-1}^{V V}= & \left(-1 / 3 l_{H}^{3}+3 / 8 l_{H}^{2}+\left(6 \zeta(2)+\frac{31}{36}\right) l_{H}-\frac{13}{4} \zeta(2)+\frac{7}{12} \zeta(3)-\frac{491}{864}\right) C_{F}{ }^{2} \\
& +\left(-\frac{5}{36} n_{f} l_{H}+\frac{1}{432}(54 \zeta(2)+65) n_{f}\right) C_{F} \\
& +\left(\left(-1 / 4 \zeta(2)+\frac{67}{72}\right) l_{H}-\frac{11}{16} \zeta(2)+\frac{13}{8} \zeta(3)-\frac{961}{864}\right) C_{F} N^{-1} \\
& +\left(1 / 12 n_{f} l_{H}-1 / 18 n_{f}\right) C_{F}+\left(1 / 8 \zeta(2)-\frac{11}{24} l_{H}+2 / 9\right) C_{F} N^{-1} \\
\mathcal{A}_{-2}^{V V}= & \left(-3 \zeta(2)-5 / 3 l_{H}+1 / 2 l_{H}^{2}+\frac{217}{144}\right) C_{F}{ }^{2} \\
\mathcal{A}_{-3}^{V V}= & \left(\frac{17}{8}-1 / 2 l_{H}\right) C_{F}{ }^{2}-1 / 8 C_{F} n_{f}+\frac{11}{16} \frac{C_{F}}{N} \\
\mathcal{A}_{-4}^{V V}= & 1 / 4 C_{F}^{2} \\
\text { with } l_{H}= & \ln \left(\frac{\mu^{2}}{m_{H}^{2}}\right) \cdot
\end{aligned}
$$




\subsection{Decay to three partons}

For the process

$$
H\left(p_{H}\right) \rightarrow b\left(p_{1}\right)+\bar{b}\left(p_{2}\right)+g\left(p_{3}\right)
$$

we require up to 1-loop corrections:

$$
\left|\mathcal{M}_{H \rightarrow b \bar{b} g}\right|^{2}=y_{b}(\mu)^{2}\left[\frac{\alpha_{s}(\mu)}{\pi} \mathcal{A}_{H \rightarrow b \bar{b} g}^{(0)}+\left(\frac{\alpha_{s}(\mu)}{\pi}\right)^{2} \mathcal{A}_{H \rightarrow b \bar{b} g}^{(1)}+\mathcal{O}\left(\alpha_{s}(\mu)^{3}\right)\right] .
$$

The tree contribution can be expressed as

$$
\mathcal{A}_{H \rightarrow b \bar{b} g}^{(0)}=m_{H}^{2} N(4 \pi)^{2}\left(\frac{\mu^{2} e^{\gamma_{E}}}{4 \pi}\right)^{\epsilon} \hat{P}_{q q}\left(\frac{s_{12}}{m_{H}^{2}}\right)\left[\frac{1}{s_{13}}+\frac{1}{s_{23}}\right]
$$

where

$$
\hat{P}_{q q}(z)=C_{F}\left[\frac{1+z^{2}}{1-z}-\epsilon(1-z)\right]
$$

is the quark gluon splitting kernel. The one-loop correction can be expressed as

$$
\begin{aligned}
& \mathcal{A}_{H \rightarrow b \bar{b} g}^{(1)}=-\frac{2 \pi^{2}\left(\mu^{2} e^{\gamma_{E}}\right)^{2 \epsilon}}{(4 \pi)^{\epsilon}} \times\left\{\left(8 \frac{\left(s_{23}+s_{13}\right)^{2} \epsilon^{2}}{s_{23} s_{13}}\right.\right. \\
& \left.+4 \frac{\left(s_{23}+s_{13}\right)^{2} \epsilon}{s_{23} s_{13}}+\frac{-12 s_{12}^{2}-12 m_{H}^{4}+8 s_{12} m_{H}^{2}}{s_{23} s_{13}}+\frac{4 s_{12}^{2}+4 m_{H}^{4}}{\epsilon s_{13} s_{23}}\right) C_{F} \operatorname{Bub}\left(s_{12}\right) \\
& +\left[\left(8+4 \frac{\left(s_{23}+s_{13}\right)\left(s_{23}+m_{H}^{2}\right) \epsilon^{2}}{\left(s_{13}+s_{12}\right) s_{23}}-4 \frac{\left(s_{12} s_{23}+3 s_{23} s_{13}-s_{23}{ }^{2}+m_{H}^{4}\right) \epsilon}{\left(s_{13}+s_{12}\right) s_{23}}\right) N C_{F}{ }^{2}\right. \\
& +\left(4 \frac{\left(s_{23}+s_{13}\right)\left(2 s_{23}+s_{13}\right) \epsilon^{2}}{s_{23} s_{13}}-4 \frac{\left(s_{12} s_{13}-s_{23}{ }^{2}-s_{13}{ }^{2}\right) \epsilon}{s_{23} s_{13}}\right. \\
& \left.\left.-4 \frac{2 s_{12}^{2}+s_{23}^{2}+s_{23} s_{13}+s_{13}^{2}+2 m_{H}^{4}}{s_{23} s_{13}}+4 \frac{s_{12}^{2}+m_{H}^{4}}{\epsilon s_{13} s_{23}}\right) C_{F}\right] \operatorname{Bub}\left(s_{23}\right) \\
& +\left[\left(8+4 \frac{\left(s_{23}+s_{13}\right)\left(s_{13}+m_{H}^{2}\right) \epsilon^{2}}{\left(s_{23}+s_{12}\right) s_{13}}-4 \frac{\left(s_{12} s_{13}+3 s_{23} s_{13}-s_{13}{ }^{2}+m_{H}^{4}\right) \epsilon}{\left(s_{23}+s_{12}\right) s_{13}}\right) N C_{F}^{2}\right. \\
& +\left(4 \frac{\left(s_{23}+2 s_{13}\right)\left(s_{23}+s_{13}\right) \epsilon^{2}}{s_{23} s_{13}}-4 \frac{\left(s_{12} s_{23}-s_{23}{ }^{2}-s_{13}{ }^{2}\right) \epsilon}{s_{23} s_{13}}\right. \\
& \left.\left.-4 \frac{2 s_{12}^{2}+s_{23}^{2}+s_{23} s_{13}+s_{13}^{2}+2 m_{H}^{4}}{s_{23} s_{13}}+4 \frac{s_{12}^{2}+m_{H}^{4}}{\epsilon s_{13} s_{23}}\right) C_{F}\right] \operatorname{Bub}\left(s_{13}\right) \\
& +\left[\left(-8 \frac{\left(s_{23}+s_{13}\right) m_{H}^{2}\left(2 s_{23} s_{13}+s_{12} s_{23}+s_{12} s_{13}\right) \epsilon^{2}}{s_{13} s_{23}\left(s_{23}+s_{12}\right)\left(s_{13}+s_{12}\right)}-8 \frac{2 s_{12}{ }^{2}+3 s_{23} s_{13}+s_{23}{ }^{2}+s_{13}{ }^{2}+2 m_{H}^{4}}{s_{23} s_{13}}\right.\right. \\
& +\frac{\left(8 m_{H}^{2}{ }^{4}-8 s_{12}{ }^{2} s_{13}{ }^{2}+16 s_{23}{ }^{2} s_{13}{ }^{2}-8 s_{23}{ }^{2} s_{12}{ }^{2}+8 s_{12}{ }^{4}-8 s_{13}{ }^{3} m_{H}^{2}-8 s_{23}{ }^{3} m_{H}^{2}\right) \epsilon}{s_{13} s_{23}\left(s_{23}+s_{12}\right)\left(s_{13}+s_{12}\right)} \\
& \left.+8 \frac{s_{12}^{2}+m_{H}^{2}{ }^{2}}{\epsilon s_{13} s_{23}}\right) N C_{F}{ }^{2}+\left(-8 \frac{\left(s_{23}+s_{13}\right)^{2} \epsilon^{2}}{s_{23} s_{13}}-4 \frac{\left(s_{23}^{2}+s_{13}^{2}\right) \epsilon}{s_{23} s_{13}}\right.
\end{aligned}
$$




$$
\begin{aligned}
& \left.\left.+4 \frac{2 s_{12}^{2}+s_{23}^{2}+s_{23} s_{13}+s_{13}^{2}+2 m_{H}^{4}}{s_{23} s_{13}}-4 \frac{s_{12}{ }^{2}+m_{H}^{4}}{\epsilon s_{13} s_{23}}\right) C_{F}\right] \operatorname{Bub}\left(m_{H}^{2}\right) \\
& +\left(2 \frac{s_{12} s_{13}\left(s_{23}+s_{13}\right) \epsilon^{2}}{s_{23}}+2 \frac{s_{12}\left(s_{23}+s_{13}\right)^{2} \epsilon}{s_{23}}-2 \frac{s_{12}\left(s_{12}^{2}+m_{H}^{4}\right)}{s_{23}}\right) C_{F} \operatorname{Box}\left(s_{12}, s_{13}, m_{H}^{2}\right) \\
& +\left(2 \frac{s_{12} s_{23}\left(s_{23}+s_{13}\right) \epsilon^{2}}{s_{13}}+2 \frac{s_{12}\left(s_{23}+s_{13}\right)^{2} \epsilon}{s_{13}}-2 \frac{s_{12}\left(s_{12}^{2}+m_{H}^{4}\right)}{s_{13}}\right) C_{F} \operatorname{Box}\left(s_{23}, s_{12}, m_{H}^{2}\right) \\
& +\left[\left(\left(-12 s_{23} s_{13}-4 s_{23}^{2}-4 s_{13}^{2}\right) \epsilon+4 s_{12}^{2}+4 m_{H}^{4}\right) C_{F}^{2}\right. \\
& \left.\left.+\left(\left(-6 s_{23} s_{13}-2 s_{23}^{2}-2 s_{13}^{2}\right) \epsilon+2 s_{12}^{2}+2 m_{H}^{4}\right) C_{F}\right] \operatorname{Box}\left(s_{13}, s_{23}, m_{H}^{2}\right)\right\} \\
& -\frac{1}{\epsilon}\left(\frac{3 C_{F}}{2}+\frac{11 C_{A}}{12}-\frac{T_{F} n_{f}}{3}\right) \mathcal{A}_{H \rightarrow b \bar{b} g}^{(0)}
\end{aligned}
$$

There are only two different master integrals which appear here. The bubble

$$
\operatorname{Bub}\left(s_{23}\right)=\int \frac{d^{d} k}{i \pi^{\frac{d}{2}}} \frac{1}{k^{2}\left(k+p_{23}\right)^{2}}=\frac{\Gamma(1+\epsilon) \Gamma(1-\epsilon)^{2}}{\epsilon \Gamma(2-2 \epsilon)}\left(-s_{23}\right)^{-\epsilon}
$$

as well as the following box integral

$$
\operatorname{Box}\left(s_{23}, s_{34}, m_{H}^{2}\right)=\int \frac{d^{d} k}{i \pi^{\frac{d}{2}}} \frac{1}{k^{2}\left(k+p_{2}\right)^{2}\left(k+p_{23}\right)^{2}\left(k+p_{234}\right)^{2}} .
$$

which can be expressed to all orders in $\epsilon$ in terms of hypergeometric functions

$$
\begin{aligned}
\operatorname{Box}\left(s, t, M^{2}\right)= & \frac{2 \Gamma(1+\epsilon) \Gamma(1-\epsilon)^{2}}{\epsilon^{2} \Gamma(1-2 \epsilon)} \frac{1}{s t}\left[-\left(-M^{2}\right)^{-\epsilon}{ }_{2} F_{1}\left(1,-\epsilon, 1-\epsilon,-\frac{u M^{2}}{s t}\right)\right. \\
& \left.+(-t)^{-\epsilon}{ }_{2} F_{1}\left(1,-\epsilon, 1-\epsilon,-\frac{u}{s}\right)+(-s)^{-\epsilon}{ }_{2} F_{1}\left(1,-\epsilon, 1-\epsilon,-\frac{u}{t}\right)\right]
\end{aligned}
$$

with $u=M^{2}-s-t$.

\subsection{Decay to four partons}

For the decay to four partons we need

$$
H\left(p_{H}\right) \rightarrow b\left(p_{1}\right)+\bar{b}\left(p_{2}\right)+i\left(p_{3}\right)+j\left(p_{4}\right), \quad(i j) \in\{(q \bar{q}),(b \bar{b}),(g g)\}
$$

at tree-level

$$
\left|\mathcal{M}_{H \rightarrow b \bar{b} i j}\right|^{2}=y_{b}(\mu)^{2}\left(\frac{\alpha_{s}(\mu)}{\pi}\right)^{2} \mathcal{A}_{H \rightarrow b \bar{b} i j}^{(0)}+\mathcal{O}\left(\alpha_{s}(\mu)^{3}\right) .
$$

The $H \rightarrow b \bar{b} q \bar{q}$ amplitude can be expressed as

$$
\mathcal{A}_{H \rightarrow b \bar{b} q \bar{q}}^{(0)}=16 \pi^{4}\left(\frac{\mu^{2} e^{\gamma_{E}}}{4 \pi}\right)^{2 \epsilon}\left[2 N C_{F} A\left(p_{1}, p_{2}, p_{3}, p_{4}\right)\right]
$$


while for two $b \bar{b}$ pairs in the final state we get

$$
\begin{aligned}
\mathcal{A}_{H \rightarrow b \bar{b} b \bar{b}}^{(0)}= & 16 \pi^{4}\left(\frac{\mu^{2} e^{\gamma_{E}}}{4 \pi}\right)^{2 \epsilon} \times \\
& \left\{2 N C_{F}\left[A\left(p_{1}, p_{2}, p_{3}, p_{4}\right)+A\left(p_{1}, p_{4}, p_{3}, p_{2}\right)+A\left(p_{3}, p_{2}, p_{1}, p_{4}\right)+A\left(p_{3}, p_{4}, p_{1}, p_{2}\right)\right]\right. \\
& \left.+2 C_{F}\left[B\left(p_{1}, p_{2}, p_{3}, p_{4}\right)+B\left(p_{3}, p_{4}, p_{1}, p_{2}\right)+B\left(p_{4}, p_{3}, p_{2}, p_{1}\right)+B\left(p_{2}, p_{1}, p_{4}, p_{3}\right)\right]\right\}
\end{aligned}
$$

where

$$
\begin{aligned}
A\left(p_{1}, p_{2}, p_{3}, p_{4}\right)= & 2\left[\frac{2(1-\epsilon)}{s_{34}}-(1+\epsilon)\left(\frac{1}{s_{134}}+\frac{1}{s_{234}}\right)+m^{2}(\epsilon-1)\left(\frac{1}{s_{134}^{2}}+\frac{1}{s_{234}^{2}}\right)\right. \\
& +\frac{s_{13}-s_{14}-2\left(s_{24}+m^{2}\right)-\epsilon\left(s_{13}+s_{14}\right)}{s_{234} s_{34}}-\frac{2 m^{2}}{s_{34}^{2}}\left(\frac{s_{14}}{s_{134}}-\frac{s_{24}}{s_{234}}\right)^{2} \quad(3.19) \\
& +\frac{s_{23}-s_{24}-2\left(s_{14}+m^{2}\right)-\epsilon\left(s_{23}+s_{24}\right)}{s_{134} s_{34}}-\frac{2 m^{2}}{s_{34}}\left(\frac{s_{14}}{s_{134}^{2}}-\frac{s_{24}}{s_{234}^{2}}\right) \\
& \left.+2 \frac{m^{4}+m^{2}\left(s_{14}+s_{24}\right)+\left(s_{14}+s_{24}\right)^{2}}{s_{134} s_{34} s_{234}}+2 \frac{(1+\epsilon) m^{2}+s_{34}+2\left(s_{14}+s_{24}\right)}{s_{134} s_{234}}\right] .
\end{aligned}
$$

The form factor $B\left(p_{1}, p_{2}, p_{3}, p_{4}\right)$ is presented in appendix A. The squared amplitude corresponding to the Higgs decaying into a $b \bar{b}$ plus two extra gluons can be expressed as

$$
\mathcal{A}_{H \rightarrow b \bar{b} g g}^{(0)}=16 \pi^{4}\left(\frac{\mu^{2} e^{\gamma_{E}}}{4 \pi}\right)^{2 \epsilon}\left\{C_{F} A_{H b \bar{b} g g}+N C_{F}^{2} B_{H b \bar{b} g g}+C_{F}\left(1+2 N C_{F}\right) C_{H b \bar{b} g g}\right\}
$$

where

$$
\begin{aligned}
& A_{H b \bar{b} g g}=A_{H b \bar{b} g g}^{(0)}+\epsilon A_{H b \bar{b} g g}^{(1)}+\epsilon^{2} A_{H b \bar{b} g g}^{(2)} \\
& B_{H b \bar{b} g g}=B_{H b \bar{b} g g}^{(0)}+\epsilon B_{H b \bar{b} g g}^{(1)}+\epsilon^{2} B_{H b \bar{b} g g}^{(2)} \\
& C_{H b \bar{b} g g}=C_{H b \bar{b} g g}^{(0)}+\epsilon C_{H b \bar{b} g g}^{(1)} .
\end{aligned}
$$

The form factors $A_{H b \bar{b} g g}^{(i)}, B_{H b \bar{b} g g}^{(i)}$ and $C_{H b \bar{b} g g}^{(i)}$ can be found in appendix A.

\section{Integration over phase space}

The matrix elements of section 3 are integrated over the phase space of the final-state partons. For convenience, we perform our calculation in the rest frame of the Higgs boson. Our results can be trivially extended to any frame of reference. The matrix-element for the $1 \rightarrow 2$ process $H \rightarrow b \bar{b}$ is independent of any phase space integration variables, and it is multiplied by the phase space volume:

$$
\Phi_{2}^{d}=\int d \Phi_{2}^{d}=\frac{1}{(2 \pi)^{d-2}} \frac{\Omega_{d-1}}{2^{d-1}}\left(m_{H}^{2}\right)^{-\epsilon}=\frac{1}{8 \pi} \frac{\left(m_{H}^{2}\right)^{-\epsilon}(4 \pi)^{\epsilon} \Gamma(1-\epsilon)}{\Gamma(2-2 \epsilon)} .
$$

The matrix elements for the $1 \rightarrow 3$ processes are integrated over the corresponding phase space with volume:

$$
\Phi_{3}=\frac{\Phi_{2}\left(m_{H}^{2}\right)^{1-2 \epsilon}}{(4 \pi)^{d / 2} \Gamma(1-\epsilon)} \int_{0}^{1} d \lambda_{1} d \lambda_{2}\left(\lambda_{1} \bar{\lambda}_{1} \lambda_{2}\right)^{-\epsilon} \bar{\lambda}_{2}^{1-2 \epsilon}
$$


The invariants take the simple form:

$$
\begin{aligned}
& s_{12}=m_{H}^{2} \lambda_{2} \\
& s_{13}=m_{H}^{2} \bar{\lambda}_{2} \lambda_{1} \\
& s_{23}=m_{H}^{2} \bar{\lambda}_{2} \bar{\lambda}_{1}
\end{aligned}
$$

In the above, we have introduced the shorthand notation

$$
\bar{\lambda} \equiv 1-\lambda .
$$

We parameterize the phase space for the $1 \rightarrow 4$ processes as follows:

$$
\Phi_{4}=N_{4} \int_{0}^{1} d \lambda_{1} d \lambda_{2} d \lambda_{3} d \lambda_{4} d \lambda_{5}\left[\lambda_{1} \bar{\lambda}_{1} \bar{\lambda}_{2}\right]^{1-2 \epsilon}\left[\lambda_{2} \lambda_{3} \bar{\lambda}_{3} \lambda_{4} \bar{\lambda}_{4}\right]^{-\epsilon}\left[\sin \left(\lambda_{5} \pi\right)\right]^{-2 \epsilon}
$$

where

$$
N_{4}=\frac{\left(m_{H}^{2}\right)^{2-3 \epsilon} 2^{-13+8 \epsilon} \pi^{-4+3 \epsilon}(1-2 \epsilon)}{\Gamma(3 / 2-\epsilon)^{2} \Gamma(1-\epsilon)} .
$$

The invariants in this parameterization are

$$
\begin{aligned}
s_{234} & =m_{H}^{2} \lambda_{1} \\
s_{34} & =m_{H}^{2} \lambda_{1} \lambda_{2} \\
s_{23} & =m_{H}^{2} \lambda_{1} \bar{\lambda}_{2} \lambda_{4} \\
s_{24} & =m_{H}^{2} \lambda_{1} \bar{\lambda}_{2} \bar{\lambda}_{4} \\
s_{12} & =m_{H}^{2} \bar{\lambda}_{1} \bar{\lambda}_{2} \bar{\lambda}_{3} \\
s_{134} & =m_{H}^{2}\left(\lambda_{2}+\lambda_{3} \bar{\lambda}_{1} \bar{\lambda}_{2}\right)
\end{aligned}
$$

and

$$
\begin{aligned}
& s_{13}=m_{H}^{2} \bar{\lambda}_{1}\left[\lambda_{4} \lambda_{3}+\lambda_{2} \bar{\lambda}_{3} \bar{\lambda}_{4}+2 \cos \left(\lambda_{5} \pi\right) \sqrt{\lambda_{2} \lambda_{3} \bar{\lambda}_{3} \lambda_{4} \bar{\lambda}_{4}}\right] \\
& s_{14}=m_{H}^{2} \bar{\lambda}_{1}\left[\lambda_{3} \bar{\lambda}_{4}+\lambda_{2} \bar{\lambda}_{3} \lambda_{4}-2 \cos \left(\lambda_{5} \pi\right) \sqrt{\lambda_{2} \lambda_{3} \bar{\lambda}_{3} \lambda_{4} \bar{\lambda}_{4}}\right] .
\end{aligned}
$$

This parameterization is very similar to the ones used in refs. [37, 57].

In the following sections we shall describe the method of non-linear mappings that we have employed in order to perform the non-trivial phase space integrations over the matrix elements at NNLO. Specifically, we shall discuss the "real-virtual" contributions which require both an integration over the three-parton phase space and an one-loop amplitude, and the "double-real" contributions which require the integration of tree matrix elements over the four-parton phase space.

\section{Real-virtual NNLO contribution}

In the one-loop amplitude for $H \rightarrow b \bar{b} g$ we will face integrals like

$$
\int d \Phi_{3} \frac{{ }_{2} F_{1}\left(1 ;-\epsilon, 1-\epsilon,-\frac{s_{23}}{s_{13}}\right)}{s_{23} s_{13}}
$$


Since the numerator in this case is not well defined for $s_{23}, s_{13} \rightarrow 0$, we can not simply apply a naive "plus-prescription" subtraction,

$$
\lambda^{-1+a \epsilon}=\frac{\delta(\lambda)}{a \epsilon}+\sum_{n=0}^{\infty} \frac{(a \epsilon)^{n}}{n !}\left[\frac{\log ^{n}(\lambda)}{\lambda}\right]_{+}
$$

to extract the singularities. To tackle this difficulty we use Euler's integral representation of the hypergeometric function, and apply a non-linear mapping to disentangle an overlapping singularity of the integration variable in the hypergeometric representation and the phase space variables which generate $s_{13}, s_{23}$. Defining

$$
F(z)=\int_{0}^{1} d x_{3} \frac{x_{3}^{-1-\epsilon}}{1+z x_{3}}=\frac{{ }_{2} F_{1}(1,-\epsilon, 1-\epsilon,-z)}{-\epsilon}
$$

we apply the transformation,

$$
x_{3} \mapsto \beta\left(x_{3}, 1, z\right), \quad \beta(x, A, B):=\frac{x A}{A+\bar{x} B},
$$

which yields

$$
F(z)=(1+z)^{\epsilon} \int_{0}^{1} d x_{3} x_{3}^{-1-\epsilon}\left(1-\frac{x_{3} z}{1+z}\right)^{\epsilon} .
$$

This representation of the hypergeometric function has finite values at the points where we need to make a subtraction,

$$
\begin{aligned}
& { }_{2} F_{1}(-\epsilon,-\epsilon, 1-\epsilon, 0)=1 \\
& { }_{2} F_{1}(-\epsilon,-\epsilon, 1-\epsilon, 1)=\Gamma(1+\epsilon) \Gamma(1-\epsilon),
\end{aligned}
$$

and we can obtain the Laurent expansion in $\epsilon$ of the real-virtual contribution easily with a "plus-prescription" subtraction. For non-special values of the kinematic variables the $\epsilon$-expansion of hypergeometric function reads:

$$
{ }_{2} F_{1}(-\epsilon,-\epsilon, 1-\epsilon, z)=\left(\frac{1}{1-z}\right)^{\epsilon}\left(1-\epsilon \log \left(\frac{1}{1-z}\right)+\sum_{k=2}^{\infty} \epsilon^{k} \operatorname{Li}_{k}\left(\frac{z}{z-1}\right)\right) .
$$

The mapping of eq. (5.4) simply re-derives a well known identity

$$
{ }_{2} F_{1}(a, b, c ; z)=(1-z)^{-b}{ }_{2} F_{1}\left(c-a, b, c ; \frac{z}{z-1}\right),
$$

and there would be no need of it had we started with the following representation of the box master integral:

$$
\begin{aligned}
\operatorname{Box}\left(u, t, M^{2}\right)= & \frac{2 \Gamma(1+\epsilon) \Gamma(1-\epsilon)^{2}}{\epsilon^{2} \Gamma(1-2 \epsilon)}(u t)^{-1-\epsilon} \\
& \times\left[-\left(-M^{2}\right)^{-\epsilon}\left(u t+s M^{2}\right)^{\epsilon}{ }_{2} F_{1}\left(-\epsilon,-\epsilon, 1-\epsilon, \frac{s M^{2}}{u t+s M^{2}}\right)\right. \\
& \left.+(-u-s)^{\epsilon}{ }_{2} F_{1}\left(-\epsilon,-\epsilon, 1-\epsilon, \frac{s}{u+s}\right)+(-t-s)^{\epsilon}{ }_{2} F_{1}\left(-\epsilon,-\epsilon, 1-\epsilon, \frac{s}{t+s}\right)\right]
\end{aligned}
$$


On the other hand, more complicated master integrals for one-loop amplitudes do not always have representations in terms of hypergeometric functions with known expansions in $\epsilon$ and variable limits. However, it is always possible to derive a Feynman representation or a hypergeometric representation (via a Mellin-Barnes representation) for them and attempt a direct numerical evaluation of the combined phase space and virtual multiple integral using non-linear transformations to factorize the singularities. We have compared the two approaches:

1. Evaluate numerically the coefficients of the Laurent expansion for the twodimensional phase space integral after we express the loop amplitude and its hypergeometric functions in terms of polylogarithms,

2. Evaluate numerically the coefficients of the Laurent expansion for the threedimensional combined phase space and loop integral.

We find that the three-dimensional integration is as fast as the two-dimensional integration where we require the evaluation of polylogarithmic functions.

\section{Integration of double-real contributions}

phase space integrals for four-parton processes develop singularities at multiple soft and collinear kinematic configurations. These singularities are overlapping. In this section, we demonstrate how they can be factorized with non-linear transformations. We begin the discussion with an integral with four singular denominators,

$$
I_{1}[J]=\int d \Phi_{4} \frac{J\left(p_{1}, p_{2}, p_{3}, p_{4}\right)}{s_{12} s_{34} s_{123} s_{234}} .
$$

where the function $J$ represents a non singular function of the partonic momenta, composed of numerators in the squared matrix elements and the infrared-safe observable $\mathcal{J}$. We disentangle the overlapping singularities using

$$
\bar{\lambda}_{3} \mapsto \alpha\left(\bar{\lambda}_{3}, \lambda_{4}, 1\right)
$$

where

$$
\alpha(x, A, B):=\frac{x A}{x A+\bar{x} B} .
$$

We note that $x \mapsto \alpha(x, A, B) \Rightarrow \bar{x} \mapsto \alpha(\bar{x}, B, A)$. After this transformation overlapping singularities still exist in the integrand but are integrable, the expansion in $\epsilon$ is therefore straightforward with simple subtractions. For the special case $J=1$, we obtain

$$
I_{1}[J=1]=N_{4}\left[61.76(2)+\frac{8.554(2)}{\epsilon}-\frac{0.1710(2)}{\epsilon^{2}}+\frac{0.34657(2)}{\epsilon^{3}}+\frac{0.25}{\epsilon^{4}}\right] .
$$

Other integrals may be mapped to $I_{1}$ of eq. (6.1) with a relabeling of the partonic momenta. For example, the integral

$$
I_{1}=\int d \Phi_{4} \frac{J\left(p_{1}, p_{2}, p_{3}, p_{4}\right)}{s_{14} s_{23} s_{134} s_{234}},
$$


which has an apparently more complicated singularity structure due to $s_{14}$ in the denominator, is transformed to the integral of eq. (6.1) by exchanging $p_{2}$ and $p_{4}$.

A second class of integrals has the following denominator structure:

$$
I_{2}[J]=\int d \Phi_{4} \frac{J\left(p_{1}, p_{2}, p_{3}, p_{4}\right)}{s_{13} s_{23} s_{134} s_{234}}
$$

Notice, that the integrand is singular not only at the boundaries of the integration variables $\lambda_{i}$, but it also develops a "line singularity" inside the integration volume. We eliminate this by casting the integral in the form

$$
I_{2}[J]=\int d \Phi_{4} \frac{\left(J\left(p_{1}, p_{2}, p_{3}, p_{4}\right)+J\left(p_{2}, p_{1}, p_{3}, p_{4}\right)\right) s_{24}}{s_{23} s_{134} s_{234}\left(s_{13} s_{24}+s_{14} s_{23}\right)} .
$$

It is easy to see that eq. (6.7) is equivalent to $I_{2}[J]$, by exchanging the momenta $p_{1} \leftrightarrow p_{2}$ in the term with $J\left(p_{2}, p_{1}, p_{3}, p_{4}\right)$. The denominator

$$
s_{13} s_{24}+s_{14} s_{23}=m_{H}^{4} \lambda_{1} \bar{\lambda}_{1} \bar{\lambda}_{2}\left[2 \lambda_{3} \lambda_{4} \bar{\lambda}_{4}+\lambda_{2} \bar{\lambda}_{3}\left(\lambda_{4}^{2}+\bar{\lambda}_{4}^{2}\right)+2 \cos \left(\pi \lambda_{5}\right)\left(1-2 \lambda_{4}\right) \sqrt{\lambda_{2} \lambda_{3} \bar{\lambda}_{3} \lambda_{4} \bar{\lambda}_{4}}\right]
$$

is only singular at the boundaries of the integration region. Applying the following sequence of mappings

$$
\begin{aligned}
& \lambda_{2} \mapsto \alpha\left(\lambda_{2}, \lambda_{3}, 1\right) \\
& \lambda_{4} \mapsto \alpha\left(\lambda_{4}, \lambda_{2} \bar{\lambda}_{3}, 1\right) \\
& \lambda_{2} \mapsto \alpha\left(\lambda_{2}, \bar{\lambda}_{1}, 1\right)
\end{aligned}
$$

leads to a factorized form. Numerically we then obtain

$$
I_{2}[J=1]=N_{4}\left[-201.16(3)-\frac{68.426(4)}{\epsilon}-\frac{12.027(8)}{\epsilon^{2}}+\frac{1.0397(1)}{\epsilon^{3}}+\frac{0.75}{\epsilon^{4}}\right] .
$$

A last class of singular integrals with four denominators is:

$$
\begin{aligned}
I_{3}[J] & =\int d \Phi_{4} \frac{J\left(p_{1}, p_{2}, p_{3}, p_{4}\right)}{s_{13} s_{23} s_{14} s_{24}} \\
& =\int d \Phi_{4} \frac{J\left(p_{1}, p_{3}, p_{2}, p_{4}\right)}{s_{12} s_{34} s_{14} s_{23}} \\
& =\int d \Phi_{4}\left(J\left(p_{1}, p_{3}, p_{2}, p_{4}\right)+J\left(p_{2}, p_{4}, p_{1}, p_{3}\right)\right) \frac{s_{24}}{s_{34} s_{12} s_{23}\left(s_{13} s_{23}+s_{14} s_{24}\right)}
\end{aligned}
$$

The term in parenthesis in the last denominator is given by

$$
s_{13} s_{23}+s_{14} s_{24}=m_{H}^{4} \lambda_{1} \bar{\lambda}_{1} \bar{\lambda}_{2}\left[2 \lambda_{2} \bar{\lambda}_{3} \lambda_{4} \bar{\lambda}_{4}+\lambda_{3}\left(\lambda_{4}^{2}+\bar{\lambda}_{4}^{2}\right)+2 \cos \left(\pi \lambda_{5}\right)\left(2 \lambda_{4}-1\right) \sqrt{\lambda_{2} \lambda_{3} \bar{\lambda}_{3} \lambda_{4} \bar{\lambda}_{4}}\right]
$$

We let $d I_{3}=\lambda_{2} d I_{3}+\bar{\lambda}_{2} d I_{3}$ and apply

$$
\begin{aligned}
\lambda_{2} & \mapsto \alpha\left(\lambda_{2}, \lambda_{3}, 1\right) \\
\lambda_{3,4} & \mapsto \alpha\left(\lambda_{3,4}, \bar{\lambda}_{2}, 1\right)
\end{aligned}
$$


to $\bar{\lambda}_{2} d I_{3}$ and

$$
\lambda_{4} \mapsto \alpha\left(\lambda_{4}, \lambda_{3}, 1\right)
$$

to $\lambda_{2} d I_{3}$. After these transformations, both integrals have only factorized singularities and can be evaluated numerically. We obtain:

$$
I_{3}[J=1]=N_{4}\left[-292.54(4)-\frac{217.030(9)}{\epsilon}-\frac{52.768(2)}{\epsilon^{2}}+\frac{6.9314(3)}{\epsilon^{3}}+\frac{5}{\epsilon^{4}}\right] .
$$

Other integrals with four denominators are mapped to $I_{1,2,3}[\mathrm{~J}]$ with simple relabeling of the partonic momenta. Integrals with fewer denominators are simpler and the non-linear mappings for them are obvious upon inspection, following the examples of ref. [74]. One case which requires special attention arises when a denominator is raised to the second power in the squares of diagrams where a gluon splits into a $q \bar{q}$ or $g g$ pair as has already been discussed in the literature (see, for example, $[68,83]$ ). These quadratic singularities are fake. A combination of terms, such as

$$
\frac{2 m_{H}^{2}}{s_{34}^{2}}\left(\frac{s_{14}}{s_{134}}-\frac{s_{24}}{s_{234}}\right)^{2}
$$

is the one which emerges in the physical matrix elements. After a non-linear mapping

$$
\lambda_{2} \rightarrow \alpha\left(\lambda_{2}, \lambda_{3}, 1\right)
$$

to factorize an overlapping singularity, the integrand is also free of quadratic singularities in the integration variables $\lambda_{i}$.

\section{$7 \quad$ Numerical results}

In this section we present our numerical results for the decay rate $\Gamma_{H \rightarrow b \bar{b} X}[\mathcal{J}]$ for selected infrared safe observables $\mathcal{J}$. We select the value of the renormalization scale $\mu=m_{H}$. First, we compute the inclusive decay width. Our numerical result is,

$$
\Gamma_{H \rightarrow b \bar{b}}^{\mathrm{NNLO}}=\Gamma_{H \rightarrow b \bar{b}}^{\mathrm{LO}}\left[1+\left(\frac{\alpha_{s}}{\pi}\right) 5.6666(4)+\left(\frac{\alpha_{s}}{\pi}\right)^{2} 29.14(2)+\mathcal{O}\left(\alpha_{s}^{3}\right)\right]
$$

with

$$
\Gamma_{H \rightarrow b \bar{b}}^{L O}=\frac{y_{b}^{2} m_{H} N}{4 \pi} .
$$

This is in agreement with the known analytic expression [1]

$$
\Gamma_{H \rightarrow b \bar{b}}^{\mathrm{NNLO}}=\Gamma_{H \rightarrow b \bar{b}}^{\mathrm{LO}}\left[1+\left(\frac{\alpha_{s}}{\pi}\right) 5.6666666 . .+\left(\frac{\alpha_{s}}{\pi}\right)^{2} 29.146714 . .+\mathcal{O}\left(\alpha_{s}^{3}\right)\right] .
$$

With our numerical program, we can compute the decay rate for arbitrary infrared-safe observables. As an example we present our results for the 2,3 and 4 jet rates using the 


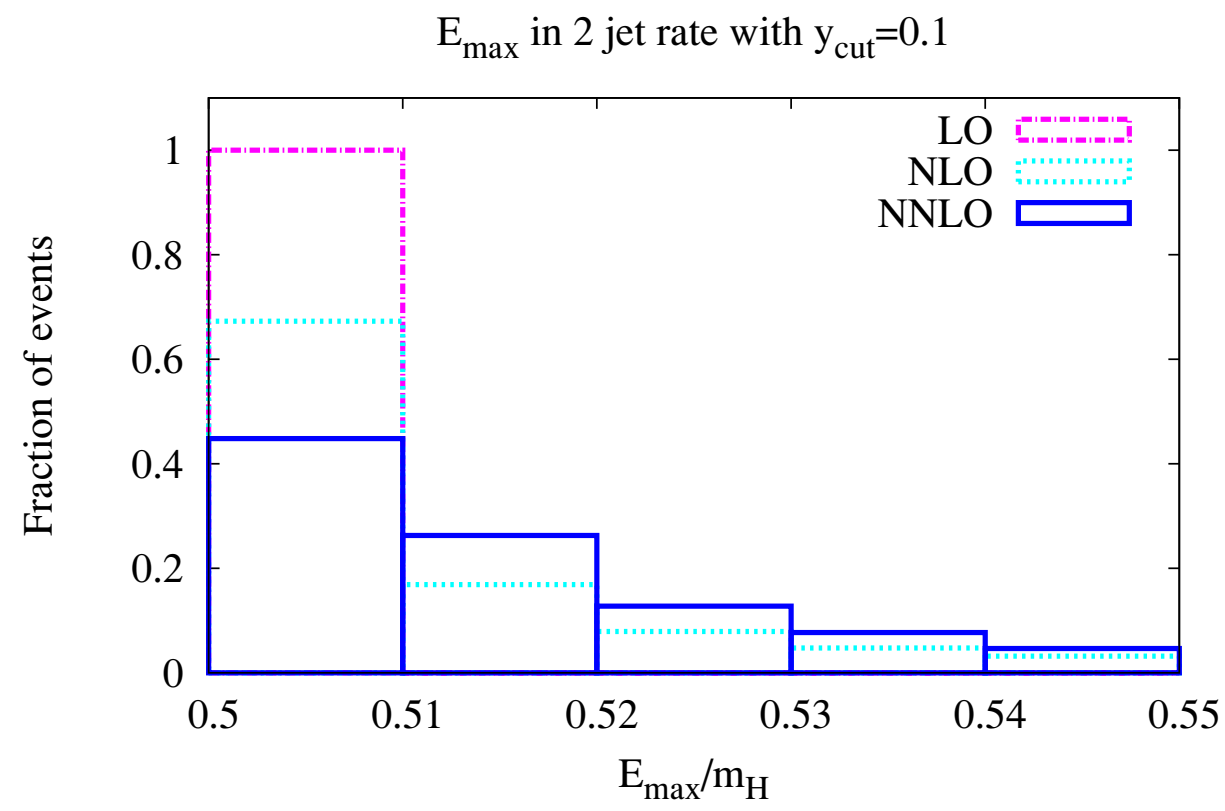

Figure 1. The energy spectrum of the leading jet in the decay $H \rightarrow b \bar{b}$ in the rest frame of the Higgs boson through NNLO. The jet clustering is performed with the JADE algorithm with $y_{\text {cut }}=0.1$.

JADE algorithm [84] with parameter $y_{\text {cut }}=0.01$ :

$$
\begin{aligned}
\Gamma_{H \rightarrow b \bar{b}}^{\mathrm{LO}}(\text { 4JetRate }) & =\Gamma_{H \rightarrow b \bar{b}}^{\mathrm{LO}}\left[+\left(\frac{\alpha_{s}}{\pi}\right)^{2} 94.1(1)+\mathcal{O}\left(\alpha_{s}^{3}\right)\right] \\
\Gamma_{H \rightarrow b \bar{b}}^{\mathrm{NLO}}(\text { 3JetRate }) & =\Gamma_{H \rightarrow b \bar{b}}^{\mathrm{LO}}\left[+\left(\frac{\alpha_{s}}{\pi}\right) 19.258(4)+\left(\frac{\alpha_{s}}{\pi}\right)^{2} 241(2)+\mathcal{O}\left(\alpha_{s}^{3}\right)\right] \\
\Gamma_{H \rightarrow b \bar{b}}^{\mathrm{NNLO}}(\text { JetRate }) & =\Gamma_{H \rightarrow b \bar{b}}^{\mathrm{LO}}\left[1-\left(\frac{\alpha_{s}}{\pi}\right) 13.591(6)-\left(\frac{\alpha_{s}}{\pi}\right)^{2} 307(2)+\mathcal{O}\left(\alpha_{s}^{3}\right)\right] .
\end{aligned}
$$

We have checked that our results for the jet-rates add up to the inclusive rate for general $y_{\text {cut }}$ values.

In figure 1 , we present the leading jet energy, $E_{\max }$, in the rest frame of the Higgs boson, for events with two jets $\left(y_{\text {cut }}=0.1\right)$. This is a new result which can only be obtained with a fully differential NNLO calculation and cannot be inferred from the inclusive decay width and NLO calculations. At leading order, $E_{\max }=\frac{m_{H}}{2}$. At higher orders, there is a range of jet energies which are kinematically allowed. We choose a value of $\alpha_{s}\left(m_{Z}\right)=0.118$ at the $\mathrm{Z}$ boson mass and evolve consistently through LO, NLO and NNLO up to the Higgs boson mass, which we assume to be $m_{H}=120 \mathrm{GeV}$.

The above numerical results demonstrate the applicability of our method to physical processes. A number of phenomenological studies which are relevant to the searches of the Higgs boson can be made. It is easy to interface our numerical code with a Monte-Carlo code for the production of a Higgs boson. We we will present complete phenomenological studies pursuing this direction in the future. 


\section{Conclusions}

In this paper, we present a first physical application of a new method for the factorization of overlapping singularities in phase space and loop integrations. We compute the fully differential decay width of a Higgs boson to a bottom-quark pair. We produce the required tree, one-loop and two-loop amplitudes with standard Feynman diagrammatic methods.

Our article focuses on the phase space integrations which emerge at NNLO. We apply non-linear mappings to factorize all overlapping singularities in all real-virtual and doublereal integrations. Consequently, we perform the expansion of all integrals in the dimension regulator $\epsilon$ with simple subtractions. The formalism allows for the computation of the decay rate for arbitrary physical observables.

We verify that we can reproduce the known results for the NNLO inclusive decay width and compute the differential two,three and four jet rates with the JADE algorithm. We also present the leading jet energy distributions, a new result that cannot be inferred from previous calculations. In the future, we will interface our NNLO Monte-Carlo to Monte-Carlo programs for the production of a Higgs boson.

We believe that our method for NNLO computations is powerful and suitable for complicated physical processes. We are looking forward to further apply our method on other interesting processes at hadron colliders.

\section{Acknowledgments}

We thank Andrea Banfi and Zoltan Kunszt for useful discussions. This research is supported by the ERC Starting Grant for the project "IterQCD" and the Swiss National Foundation under contract SNF 200020-126632.

\section{A Double-real matrix elements}

The function $B\left(p_{1}, p_{2}, p_{3}, p_{4}\right)$ occurring in the $H \rightarrow b \bar{b} b \bar{b}$ squared amplitude can be expressed as

$$
\begin{aligned}
& B\left(p_{1}, p_{2}, p_{3}, p_{4}\right)=2 s_{34}{ }^{-1}-2 s_{134}{ }^{-1}-4 s_{234}{ }^{-1}+2 s_{124}{ }^{-1}-2 \frac{s_{12}\left(s_{13}{ }^{2}+2 s_{12}{ }^{2}+2 s_{12} s_{13}\right)}{s_{14} s_{34} s_{124} s_{234}} \\
& +4 \frac{s_{12} s_{23}}{s_{34} s_{134} s_{234}}-2 \frac{s_{12}\left(s_{23}+2 s_{13}+s_{12}\right)}{s_{14} s_{34} s_{124}}+4 \frac{s_{12} s_{23}}{s_{14} s_{124} s_{134}}+ \\
& \left(\frac{4 s_{13} s_{14}+2 s_{13}^{2}+4 s_{12} s_{13}+2 s_{14}^{2}+2 s_{12} s_{14}}{s_{34} s_{124} s_{234}}+\frac{-2 s_{24}-4 s_{13}}{s_{14} s_{34}}\right. \\
& +\frac{-2 s_{24}+2 s_{12}-2 s_{23}}{s_{14} s_{234}}+\frac{4 s_{14}-4 s_{23}}{s_{124} s_{134}}+\frac{-4 s_{12}-4 s_{14}-4 s_{13}}{s_{134} s_{234}} \\
& +\frac{2 s_{14}+4 s_{13}+2 s_{23}}{s_{34} s_{124}}+2 \frac{s_{12} s_{13}}{s_{14} s_{34} s_{124}}+\frac{-2 s_{24}+2 s_{12}}{s_{14} s_{124}}-2 \frac{s_{13}\left(-s_{23}+s_{13}\right)}{s_{14} s_{34} s_{234}} \\
& +\frac{-4 s_{13}-2 s_{14}+2 s_{23}}{s_{34} s_{234}}+\frac{4 s_{12} s_{13}+2 s_{24} s_{23}+2 s_{24}^{2}}{s_{14} s_{124} s_{234}}+\frac{-4 s_{12}-4 s_{24}-4 s_{23}}{s_{134}{ }^{2}} \\
& +\frac{2 s_{24}+2 s_{23}}{s_{34} s_{134}}+2 s_{14}{ }^{-1}+2 \frac{s_{12} s_{13}{ }^{2}}{s_{14} s_{34} s_{124} s_{234}}-8 s_{134}{ }^{-1}+2 s_{234}{ }^{-1}+\frac{2 s_{12}+2 s_{24}}{s_{14} s_{134}}
\end{aligned}
$$




$$
\begin{aligned}
& \left.-2 s_{124}{ }^{-1}\right) \epsilon^{2}+\left(-4 s_{14}^{-1}+6 s_{34}^{-1}+6 s_{134}^{-1}+4 s_{234}^{-1}-2 s_{124}{ }^{-1}\right. \\
& +2 \frac{s_{12} s_{13}{ }^{2}}{s_{14} s_{34} s_{124} s_{234}}-4 \frac{s_{12} s_{23}}{s_{34} s_{134} s_{234}}-4 \frac{s_{12} s_{23}}{s_{14} s_{124} s_{134}} \\
& -2 \frac{s_{12}\left(-s_{23}-2 s_{13}+s_{12}\right)}{s_{14} s_{34} s_{124}}+\frac{4 s_{12}+4 s_{24}+4 s_{23}}{s_{134}{ }^{2}} \\
& +\frac{-6 s_{24}-8 s_{13}}{s_{14} s_{34}}+\frac{-6 s_{12}-2 s_{23}+6 s_{34}+8 s_{13}}{s_{14} s_{134}}+\frac{6 s_{13}+6 s_{24}-2 s_{14}+2 s_{23}}{s_{34} s_{134}} \\
& +\frac{6 s_{13}+4 s_{24}+10 s_{12}}{s_{14} s_{234}}+\frac{-2 s_{23}-4 s_{24}+2 s_{14}+4 s_{12}}{s_{34} s_{234}}+\frac{-4 s_{13}-2 s_{14}-4 s_{12}}{s_{134} s_{234}} \\
& +\frac{-2 s_{24}-2 s_{12}}{s_{14} s_{124}}+\frac{6 s_{23}-8 s_{12}-8 s_{14}+6 s_{13}}{s_{34} s_{124}}+\frac{-2 s_{13}-4 s_{23}+2 s_{14}}{s_{124} s_{134}} \\
& +\frac{-4 s_{14}-4 s_{13}-8 s_{12}}{s_{124} s_{234}}+4 \frac{s_{13}\left(s_{12}+s_{24}+s_{23}\right)}{s_{14} s_{134}{ }^{2}}+4 \frac{s_{13}\left(s_{12}+s_{24}+s_{23}\right)}{s_{34} s_{134}{ }^{2}} \\
& +\frac{4 s_{13} s_{23}+2 s_{12} s_{23}-2 s_{13}^{2}-2 s_{23}^{2}}{s_{14} s_{34} s_{234}} \\
& +\frac{2 s_{12}^{2}+4 s_{23}^{2}+8 s_{24} s_{23}+4 s_{12} s_{23}+6 s_{24}^{2}+8 s_{12} s_{24}}{s_{14} s_{234} s_{134}} \\
& +\frac{-4 s_{12} s_{23}+6 s_{14}^{2}+4 s_{12} s_{14}+2 s_{23}^{2}+2 s_{12}^{2}-8 s_{23} s_{14}}{s_{34} s_{124} s_{134}}+\frac{2 s_{24}^{2}+2 s_{24} s_{23}-4 s_{12}{ }^{2}}{s_{14} s_{124} s_{234}} \\
& \left.+\frac{-4 s_{12}^{2}-6 s_{12} s_{14}+2 s_{13}^{2}-2 s_{14}^{2}}{s_{34} s_{124} s_{234}}\right) \epsilon+\frac{4 s_{12}+4 s_{23}+8 s_{13}}{s_{14} s_{34}} \\
& +\frac{-6 s_{13}-2 s_{14}-4 s_{24}}{s_{34} s_{134}}+\frac{2 s_{23}-2 s_{24}+4 s_{12}-2 s_{34}-4 s_{13}}{s_{14} s_{134}} \\
& +\frac{-4 s_{23}-10 s_{12}+4 s_{24}-6 s_{13}}{s_{14} s_{234}}+\frac{4 s_{12}+4 s_{13}+2 s_{23}+2 s_{14}}{s_{34} s_{234}} \\
& +\frac{2 s_{14}+4 s_{13}+4 s_{12}}{s_{134} s_{234}}+\frac{2 s_{12}+2 s_{24}}{s_{14} s_{124}}+\frac{-6 s_{13}-6 s_{23}-4 s_{12}}{s_{34} s_{124}}+\frac{2 s_{13}-2 s_{14}+4 s_{23}}{s_{124} s_{134}} \\
& +\frac{4 s_{14}+4 s_{13}+8 s_{12}}{s_{124} s_{234}}-4 \frac{s_{13}\left(s_{12}+s_{24}+s_{23}\right)}{s_{14} s_{134}{ }^{2}}-4 \frac{s_{13}\left(s_{12}+s_{24}+s_{23}\right)}{s_{34} s_{134}{ }^{2}} \\
& +\frac{-2 s_{23}^{2}-2 s_{12} s_{23}+2 s_{13}^{2}-4 s_{13} s_{23}+4 s_{12}^{2}+4 s_{24}^{2}+4 s_{12} s_{13}-4 s_{24} s_{13}-4 s_{12} s_{24}}{s_{14} s_{34} s_{234}} \\
& +\frac{-2 s_{12}{ }^{2}-2 s_{24}^{2}-4 s_{23}{ }^{2}-4 s_{12} s_{24}-4 s_{24} s_{23}-4 s_{12} s_{23}}{s_{14} s_{234} s_{134}} \\
& +\frac{-2 s_{23}^{2}-2 s_{12}^{2}+4 s_{23} s_{14}-2 s_{14}^{2}}{s_{34} s_{124} s_{134}}+\frac{4 s_{12}^{2}-2 s_{24} s_{23}-2 s_{24}^{2}}{s_{14} s_{124} s_{234}} \\
& +\frac{-4 s_{13} s_{14}-8 s_{12}^{2}-2 s_{13}^{2}-2 s_{14}^{2}-8 s_{12} s_{13}-6 s_{12} s_{14}}{s_{34} s_{124} s_{234}}
\end{aligned}
$$

$$
\begin{aligned}
A_{H b \bar{b} g g}^{(0)}= & 20 s_{234}{ }^{-1}+20 s_{134}{ }^{-1}+32 s_{13}{ }^{-1}+24 s_{24}{ }^{-1}+24 s_{14}{ }^{-1}+32 s_{23}{ }^{-1} \\
& +4 \frac{3 s_{13}-6 m_{H}{ }^{2}+3 s_{14}+6 s_{34}}{s_{24} s_{23}}+8 \frac{3 s_{24}-6 m_{H}^{2}+3 s_{14}+3 s_{34}}{s_{13} s_{23}} \\
& +4 \frac{6 m_{H}{ }^{2}-3 s_{13}+3 s_{34}+3 s_{24}}{s_{234} s_{14}}+4 \frac{-s_{24}-s_{14}+s_{34}+4 m_{H}{ }^{2}}{s_{234} s_{13}}
\end{aligned}
$$




$$
\begin{aligned}
& -4 \frac{-3 s_{14}-6 m_{H}^{2}+3 s_{23}-3 s_{34}}{s_{134} s_{24}}+4 \frac{3 s_{23}+6 s_{34}-6 m_{H}^{2}+3 s_{24}}{s_{13} s_{14}} \\
& +8 \frac{-5 s_{34}-8 m_{H}^{2}-4 s_{24}-4 s_{14}}{s_{134} s_{234}}+8 \frac{m_{H}^{2}}{s_{134}{ }^{2}}+8 \frac{m_{H}^{2}}{s_{234}{ }^{2}} \\
& -4 \frac{8 m_{H}^{2}-5 s_{14}-7 s_{34}-5 s_{23}}{s_{24} s_{13}}+4 \frac{-s_{24}-s_{14}+s_{34}+4 m_{H}^{2}}{s_{134} s_{23}} \\
& -4 \frac{-5 s_{24}+8 m_{H}^{2}-5 s_{13}-7 s_{34}}{s_{14} s_{23}}-4 \frac{-s_{14}-2 s_{34}+s_{13}-3 m_{H}^{2}}{s_{234} s_{24}} \\
& +8 \frac{3 s_{23}+3 s_{13}+3 s_{34}-6 m_{H}^{2}}{s_{24} s_{14}}-4 \frac{-3 m_{H}^{2}-2 s_{34}-s_{24}+s_{23}}{s_{134} s_{14}} \\
& +4 \frac{\left(m_{H}^{2}+s_{34}\right)\left(-2 m_{H}^{4}-s_{34}^{2}-2 m_{H}^{2} s_{34}\right)}{s_{234} s_{134} s_{13} s_{23}} \\
& +4 \frac{\left(m_{H}^{2}+s_{34}\right)\left(-2 m_{H}^{4}-s_{34}^{2}-2 m_{H}^{2} s_{34}\right)}{s_{234} s_{134} s_{14} s_{24}} \\
& +4 \frac{\left(-s_{34}+m_{H}^{2}\right)\left(-2 m_{H}^{4}-s_{34}^{2}+2 m_{H}^{2} s_{34}\right)}{s_{13} s_{14} s_{23} s_{24}} \\
& -4 \frac{3 m_{H}^{2} s_{13}-s_{34}^{2}-s_{13}^{2}-4 m_{H}^{4}-3 m_{H}^{2} s_{34}+s_{13} s_{34}}{s_{234} s_{24} s_{14}} \\
& -4 \frac{-s_{34}{ }^{2}-4 m_{H}{ }^{4}-s_{24}{ }^{2}+s_{24} s_{34}+3 m_{H}{ }^{2} s_{24}-3 m_{H}{ }^{2} s_{34}}{s_{134} s_{13} s_{23}} \\
& -4 \frac{3 m_{H}^{2} s_{14}-s_{14}^{2}+s_{14} s_{34}-3 m_{H}^{2} s_{34}-4 m_{H}^{4}-s_{34}^{2}}{s_{234} s_{13} s_{23}} \\
& -4 \frac{s_{23} s_{34}-4 m_{H}{ }^{4}-3 m_{H}^{2} s_{34}-s_{34}{ }^{2}-s_{23}{ }^{2}+3 m_{H}{ }^{2} s_{23}}{s_{134} s_{14} s_{24}} \\
& +4 \frac{-s_{34}{ }^{2}-4 m_{H}^{4}-s_{24}{ }^{2}+s_{24} s_{34}+3 m_{H}^{2} s_{24}-3 m_{H}^{2} s_{34}}{s_{134} s_{13} s_{234}} \\
& +4 \frac{-s_{24}{ }^{2}-3 s_{24} s_{34}-4 m_{H}{ }^{4}-3 s_{34}{ }^{2}-6 m_{H}{ }^{2} s_{34}-3 m_{H}{ }^{2} s_{24}}{s_{234} s_{134} s_{14}} \\
& +4 \frac{-3 s_{34}^{2}-3 s_{14} s_{34}-4 m_{H}^{4}-3 m_{H}^{2} s_{14}-s_{14}^{2}-6 m_{H}^{2} s_{34}}{s_{134} s_{234} s_{24}} \\
& +4 \frac{3 m_{H}^{2} s_{14}-s_{14}^{2}+s_{14} s_{34}-3 m_{H}^{2} s_{34}-4 m_{H}^{4}-s_{34}^{2}}{s_{234} s_{134} s_{23}} \\
& +4 \frac{4 m_{H}^{4}+s_{23}^{2}+3 s_{34}^{2}-3 m_{H}^{2} s_{23}-6 m_{H}^{2} s_{34}+3 s_{23} s_{34}}{s_{24} s_{13} s_{14}} \\
& +4 \frac{4 m_{H}^{4}+3 s_{34}^{2}+s_{24}{ }^{2}-6 m_{H}{ }^{2} s_{34}+3 s_{24} s_{34}-3 m_{H}^{2} s_{24}}{s_{13} s_{14} s_{23}} \\
& +4 \frac{-3 m_{H}^{2} s_{14}+4 m_{H}^{4}+s_{14}^{2}+3 s_{14} s_{34}+3 s_{34}^{2}-6 m_{H}^{2} s_{34}}{s_{24} s_{13} s_{23}} \\
& +4 \frac{-6 m_{H}^{2} s_{34}+4 m_{H}^{4}+3 s_{13} s_{34}+s_{13}^{2}-3 m_{H}^{2} s_{13}+3 s_{34}^{2}}{s_{24} s_{14} s_{23}}
\end{aligned}
$$




$$
\begin{aligned}
& A_{H b \bar{b} g g}^{(1)}=-16 s_{234}{ }^{-1}-32 s_{13}{ }^{-1}-24 s_{14}{ }^{-1}-16 s_{134}{ }^{-1}-24 s_{24}{ }^{-1}-32 s_{23}{ }^{-1} \\
& -16 \frac{m_{H}^{2}}{s_{234}{ }^{2}}-16 \frac{m_{H}^{2}}{s_{134}{ }^{2}}-4 \frac{s_{14}+2 s_{34}}{s_{234} s_{24}}-4 \frac{s_{24}+2 s_{34}}{s_{134} s_{14}} \\
& +8 \frac{-3 s_{24}-3 s_{34}+2 m_{H}^{2}-3 s_{14}}{s_{13} s_{23}}+4 \frac{-5 s_{34}-2 s_{13}+m_{H}^{2}-2 s_{14}}{s_{24} s_{23}} \\
& +4 \frac{-3 m_{H}^{2}-s_{34}+s_{14}+s_{24}}{s_{134} s_{23}}+4 \frac{-3 s_{24}-3 s_{34}-3 m_{H}^{2}+4 s_{13}}{s_{234} s_{14}} \\
& -4 \frac{-3 m_{H}^{2}+8 s_{34}+6 s_{23}+6 s_{14}}{s_{24} s_{13}}-4 \frac{3 m_{H}^{2}+3 s_{34}+3 s_{14}-4 s_{23}}{s_{134} s_{24}} \\
& +4 \frac{-3 m_{H}^{2}-s_{34}+s_{14}+s_{24}}{s_{234} s_{13}}-4 \frac{-3 m_{H}^{2}+8 s_{34}+6 s_{13}+6 s_{24}}{s_{14} s_{23}} \\
& +8 \frac{5 s_{34}+4 m_{H}^{2}+4 s_{14}+4 s_{24}}{s_{134} s_{234}}-4 \frac{-m_{H}^{2}+s_{14}}{s_{234} s_{23}}-4 \frac{-m_{H}^{2}+s_{24}}{s_{134} s_{13}} \\
& +8 \frac{2 m_{H}^{2}-3 s_{23}-3 s_{13}-3 s_{34}}{s_{24} s_{14}}+4 \frac{-2 s_{24}-5 s_{34}-2 s_{23}+m_{H}{ }^{2}}{s_{13} s_{14}} \\
& -4 \frac{s_{13}{ }^{2}-m_{H}{ }^{2} s_{13}-s_{13} s_{34}+m_{H}{ }^{2} s_{34}+s_{34}{ }^{2}}{s_{234} s_{24} s_{14}} \\
& -4 \frac{s_{24}{ }^{2}-m_{H}{ }^{2} s_{24}-s_{24} s_{34}+s_{34}{ }^{2}+m_{H}{ }^{2} s_{34}}{s_{134} s_{13} s_{23}} \\
& -4 \frac{-s_{14} s_{34}+m_{H}{ }^{2} s_{34}+s_{34}{ }^{2}-m_{H}^{2} s_{14}+s_{14}{ }^{2}}{s_{234} s_{13} s_{23}} \\
& -4 \frac{m_{H}^{2} s_{34}+s_{23}^{2}-s_{23} s_{34}-m_{H}{ }^{2} s_{23}+s_{34}^{2}}{s_{134} s_{14} s_{24}} \\
& +4 \frac{-s_{14} s_{34}+m_{H}{ }^{2} s_{34}+s_{34}{ }^{2}-m_{H}^{2} s_{14}+s_{14}{ }^{2}}{s_{234} s_{134} s_{23}} \\
& +4 \frac{s_{24}{ }^{2}-m_{H}{ }^{2} s_{24}-s_{24} s_{34}+s_{34}{ }^{2}+m_{H}^{2} s_{34}}{s_{134} s_{13} s_{234}} \\
& +4 \frac{s_{24}{ }^{2}+3 s_{34}^{2}+m_{H}^{2} s_{24}+3 s_{24} s_{34}+2 m_{H}^{2} s_{34}}{s_{234} s_{134} s_{14}} \\
& +4 \frac{2 m_{H}^{2} s_{34}+m_{H}^{2} s_{14}+s_{14}^{2}+3 s_{34}^{2}+3 s_{14} s_{34}}{s_{134} s_{234} s_{24}} \\
& +4 \frac{-s_{13}{ }^{2}+2 m_{H}{ }^{2} s_{34}+m_{H}{ }^{2} s_{13}-3 s_{13} s_{34}-3 s_{34}{ }^{2}}{s_{24} s_{14} s_{23}} \\
& +4 \frac{-3 s_{23} s_{34}+m_{H}^{2} s_{23}-s_{23}^{2}-3 s_{34}^{2}+2 m_{H}^{2} s_{34}}{s_{24} s_{13} s_{14}} \\
& +4 \frac{m_{H}^{2} s_{24}+2 m_{H}^{2} s_{34}-3 s_{24} s_{34}-s_{24}^{2}-3 s_{34}^{2}}{s_{13} s_{14} s_{23}} \\
& +4 \frac{-3 s_{34}^{2}-s_{14}^{2}+m_{H}^{2} s_{14}+2 m_{H}^{2} s_{34}-3 s_{14} s_{34}}{s_{24} s_{13} s_{23}} \\
& +4 \frac{\left(-s_{34}+m_{H}^{2}\right) s_{34}{ }^{2}}{s_{13} s_{14} s_{23} s_{24}}+4 \frac{\left(m_{H}^{2}+s_{34}\right) s_{34}^{2}}{s_{234} s_{134} s_{13} s_{23}}+4 \frac{\left(m_{H}^{2}+s_{34}\right) s_{34}^{2}}{s_{234} s_{134} s_{14} s_{24}}
\end{aligned}
$$




$$
\begin{aligned}
& A_{H b \bar{b} g g}^{(2)}=-4 s_{23}{ }^{-1}-4 s_{13}{ }^{-1}-8 s_{134}{ }^{-1}-8 s_{234}{ }^{-1} \\
& +8 \frac{m_{H}^{2}}{s_{134}{ }^{2}}+8 \frac{m_{H}^{2}}{s_{234}{ }^{2}}+8 \frac{2 m_{H}^{2}+2 s_{34}}{s_{134} s_{234}}-4 \frac{s_{34}+m_{H}^{2}-s_{23}}{s_{134} s_{14}} \\
& -4 \frac{s_{34}-s_{13}+m_{H}^{2}}{s_{234} s_{24}}+4 \frac{-2 s_{34}+s_{13}-s_{24}}{s_{234} s_{14}}-4 \frac{3 s_{34}+s_{14}+s_{23}}{s_{24} s_{13}} \\
& +4 \frac{s_{14}+s_{24}-s_{34}}{s_{134} s_{23}}-4 \frac{s_{14}+2 s_{34}-s_{23}}{s_{134} s_{24}}+4 \frac{s_{14}+s_{24}-s_{34}}{s_{234} s_{13}} \\
& -4 \frac{s_{24}+3 s_{34}+s_{13}}{s_{14} s_{23}}-4 \frac{m_{H}^{2}-s_{14}}{s_{234} s_{23}}-4 \frac{m_{H}^{2}-s_{24}}{s_{134} s_{13}} \\
& +4 \frac{-s_{13}+m_{H}^{2}-3 s_{34}-s_{14}}{s_{24} s_{23}}+4 \frac{-s_{24}-3 s_{34}+m_{H}^{2}-s_{23}}{s_{13} s_{14}} \\
& -4 \frac{m_{H}^{2} s_{34}-s_{13} s_{34}+s_{34}^{2}}{s_{234} s_{24} s_{14}}-4 \frac{s_{34}^{2}-s_{24} s_{34}+m_{H}^{2} s_{34}}{s_{134} s_{13} s_{23}} \\
& -4 \frac{s_{34}{ }^{2}+m_{H}{ }^{2} s_{34}-s_{14} s_{34}}{s_{234} s_{13} s_{23}}-4 \frac{-s_{23} s_{34}+m_{H}{ }^{2} s_{34}+s_{34}{ }^{2}}{s_{134} s_{14} s_{24}} \\
& +4 \frac{s_{34}{ }^{2}-s_{24} s_{34}+m_{H}{ }^{2} s_{34}}{s_{134} s_{13} s_{234}}+4 \frac{s_{24} s_{34}+m_{H}^{2} s_{34}+2 s_{34}{ }^{2}}{s_{234} s_{134} s_{14}} \\
& +4 \frac{s_{14} s_{34}+m_{H}^{2} s_{34}+2 s_{34}^{2}}{s_{134} s_{234} s_{24}}+4 \frac{s_{34}^{2}+m_{H}^{2} s_{34}-s_{14} s_{34}}{s_{234} s_{134} s_{23}} \\
& +4 \frac{-2 s_{34}^{2}-s_{23} s_{34}+m_{H}^{2} s_{34}}{s_{24} s_{13} s_{14}}+4 \frac{-s_{24} s_{34}+m_{H}^{2} s_{34}-2 s_{34}^{2}}{s_{13} s_{14} s_{23}} \\
& +4 \frac{-2 s_{34}^{2}-s_{14} s_{34}+m_{H}^{2} s_{34}}{s_{24} s_{13} s_{23}}+4 \frac{-2 s_{34}{ }^{2}-s_{13} s_{34}+m_{H}{ }^{2} s_{34}}{s_{24} s_{14} s_{23}} \\
& +4 \frac{\left(-s_{34}+m_{H}{ }^{2}\right) s_{34}{ }^{2}}{s_{13} s_{14} s_{23} s_{24}}+4 \frac{\left(m_{H}^{2}+s_{34}\right) s_{34}{ }^{2}}{s_{234} s_{134} s_{13} s_{23}}+4 \frac{\left(m_{H}^{2}+s_{34}\right) s_{34}{ }^{2}}{s_{234} s_{134} s_{14} s_{24}}
\end{aligned}
$$

$$
\begin{aligned}
& B_{H b \bar{b} g g}^{(0)}=8 s_{14}{ }^{-1}+16 s_{23}{ }^{-1}+8 s_{24}{ }^{-1}+16 s_{13}{ }^{-1}+40 s_{134}{ }^{-1}+40 s_{234}{ }^{-1} \\
& +8 \frac{m_{H}^{2}\left(-2 m_{H}^{2}+s_{34}+s_{14}\right)}{s_{234} s_{24} s_{13}}+8 \frac{m_{H}^{2}\left(-2 m_{H}^{2}+s_{34}+s_{24}\right)}{s_{134} s_{14} s_{23}} \\
& -8 \frac{m_{H}^{2}\left(2 m_{H}^{2}-s_{13}+s_{24}\right)}{s_{234} s_{14} s_{23}}-8 \frac{m_{H}^{2}\left(2 m_{H}^{2}+s_{14}-s_{23}\right)}{s_{134} s_{13} s_{24}} \\
& +16 \frac{m_{H}^{6}}{s_{134} s_{13} s_{234} s_{24}}+16 \frac{m_{H}^{6}}{s_{234} s_{134} s_{14} s_{23}}+8 \frac{s_{14}+2 m_{H}^{2}}{s_{234} s_{13}}+8 \frac{m_{H}^{2}}{s_{134} s_{13}} \\
& -8 \frac{-3 m_{H}^{2}+s_{23}-s_{24}-s_{34}}{s_{134} s_{14}}+8 \frac{2 s_{13}+s_{34}+2 s_{24}-m_{H}{ }^{2}}{s_{14} s_{23}} \\
& -8 \frac{s_{13}-s_{34}-3 m_{H}^{2}-s_{14}}{s_{234} s_{24}}+8 \frac{m_{H}^{2}}{s_{234} s_{23}}-8 \frac{m_{H}^{2} s_{24}}{s_{23} s_{234}{ }^{2}}+8 \frac{m_{H}^{2} s_{34}}{s_{134}{ }^{2} s_{14}} \\
& +8 \frac{m_{H}^{2} s_{34}}{s_{234^{2}} s_{24}}-8 \frac{m_{H}^{2} s_{14}}{s_{134^{2}} s_{13}}+8 \frac{-s_{24}^{2}-4 m_{H}^{4}+3 m_{H}^{2} s_{24}}{s_{134} s_{13} s_{234}} \\
& +8 \frac{-2 s_{24} s_{34}-3 m_{H}^{2} s_{34}-4 m_{H}^{4}-s_{24}{ }^{2}-s_{34}{ }^{2}-3 m_{H}{ }^{2} s_{24}}{s_{234} s_{134} s_{14}} \\
& +8 \frac{-3 m_{H}^{2} s_{34}-s_{14}{ }^{2}-2 s_{14} s_{34}-s_{34}{ }^{2}-4 m_{H}^{4}-3 m_{H}{ }^{2} s_{14}}{s_{134} s_{234} s_{24}}
\end{aligned}
$$




$$
\begin{aligned}
& +8 \frac{3 m_{H}^{2} s_{14}-4 m_{H}^{4}-s_{14}^{2}}{s_{234} s_{134} s_{23}}+24 \frac{m_{H}^{2}}{s_{134^{2}}}+24 \frac{m_{H}^{2}}{s_{234}{ }^{2}} \\
& -8 \frac{-s_{34}+s_{13}-3 m_{H}^{2}-2 s_{24}}{s_{234} s_{14}}+8 \frac{-m_{H}^{2}+s_{34}+2 s_{14}+2 s_{23}}{s_{24} s_{13}} \\
& +8 \frac{s_{24}+2 m_{H}^{2}}{s_{134} s_{23}}-8 \frac{-s_{34}-2 s_{14}-3 m_{H}^{2}+s_{23}}{s_{134} s_{24}} \\
& +16 \frac{-6 m_{H}^{2}-4 s_{24}-4 s_{14}-3 s_{34}}{s_{134} s_{234}} \\
& B_{H b \bar{b} g g}^{(1)}=-8 s_{14}{ }^{-1}-16 s_{23}{ }^{-1}-8 s_{24}{ }^{-1}-16 s_{13}{ }^{-1}-48 s_{134}{ }^{-1}-48 s_{234}{ }^{-1} \\
& +8 \frac{m_{H}^{2}\left(-2 s_{34}-s_{14}\right)}{s_{234} s_{24} s_{13}}+8 \frac{m_{H}^{2}\left(-2 s_{34}-s_{24}\right)}{s_{134} s_{14} s_{23}}-8 \frac{m_{H}^{2}\left(s_{13}-2 s_{24}\right)}{s_{234} s_{14} s_{23}} \\
& -8 \frac{m_{H}^{2}\left(-2 s_{14}+s_{23}\right)}{s_{134} s_{13} s_{24}}+8 \frac{s_{13}}{s_{234} s_{23}}-8 \frac{-m_{H}^{2}+s_{34}-s_{23}}{s_{134} s_{14}} \\
& -8 \frac{-s_{13}-m_{H}^{2}+s_{34}}{s_{234} s_{24}}+16 \frac{m_{H}^{2} s_{24}}{s_{23} s_{234}}-16 \frac{m_{H}^{2} s_{34}}{s_{134}{ }^{2} s_{14}} \\
& -16 \frac{m_{H}^{2} s_{34}}{s_{234^{2}} s_{24}}+16 \frac{m_{H}^{2} s_{14}}{s_{134^{2}} s_{13}}-32 \frac{m_{H}^{2}}{s_{134}{ }^{2}}-32 \frac{m_{H}^{2}}{s_{234}{ }^{2}} \\
& +8 \frac{s_{24}{ }^{2}-m_{H}^{2} s_{24}}{s_{134} s_{13} s_{234}}+8 \frac{2 s_{24} s_{34}+s_{34}{ }^{2}+s_{24}{ }^{2}+m_{H}^{2} s_{24}+m_{H}{ }^{2} s_{34}}{s_{234} s_{134} s_{14}} \\
& +8 \frac{s_{34}^{2}+2 s_{14} s_{34}+m_{H}^{2} s_{14}+s_{14}^{2}+m_{H}^{2} s_{34}}{s_{134} s_{234} s_{24}}+8 \frac{s_{14}{ }^{2}-m_{H}{ }^{2} s_{14}}{s_{234} s_{134} s_{23}} \\
& -8 \frac{-s_{13}+m_{H}^{2}+2 s_{24}+s_{34}}{s_{234} s_{14}}+8 \frac{-2 s_{14}+m_{H}^{2}-s_{34}-2 s_{23}}{s_{24} s_{13}} \\
& +8 \frac{-2 s_{24}-3 m_{H}^{2}}{s_{134} s_{23}}+8 \frac{s_{23}}{s_{134} s_{13}}-8 \frac{m_{H}^{2}-s_{23}+s_{34}+2 s_{14}}{s_{134} s_{24}} \\
& +8 \frac{-3 m_{H}^{2}-2 s_{14}}{s_{234} s_{13}}+8 \frac{-s_{34}-2 s_{24}-2 s_{13}+m_{H}^{2}}{s_{14} s_{23}} \\
& +16 \frac{4 s_{14}+4 s_{24}+2 m_{H}^{2}+3 s_{34}}{s_{134} s_{234}} \\
& B_{H b \bar{b} g g}^{(2)}=-8 \frac{s_{34}+s_{24}}{s_{134} s_{14}}-8 s_{23}{ }^{-1}-8 s_{13}{ }^{-1}-16 s_{134}{ }^{-1}-16 s_{234}{ }^{-1} \\
& +8 \frac{m_{H}^{2} s_{34}}{s_{234} s_{24} s_{13}}+8 \frac{m_{H}^{2} s_{34}}{s_{134} s_{14} s_{23}}-8 \frac{m_{H}^{2} s_{24}}{s_{234} s_{14} s_{23}}-8 \frac{m_{H}^{2} s_{14}}{s_{134} s_{13} s_{24}} \\
& +8 \frac{-s_{13}+m_{H}^{2}}{s_{234} s_{23}}-8 \frac{s_{34}+s_{14}}{s_{234} s_{24}}-8 \frac{m_{H}^{2} s_{24}}{s_{23} s_{234}{ }^{2}} \\
& +8 \frac{m_{H}^{2} s_{34}}{s_{134}{ }^{2} s_{14}}+8 \frac{m_{H}^{2} s_{34}}{s_{234}{ }^{2} s_{24}}-8 \frac{m_{H}^{2} s_{14}}{s_{134}{ }^{2} s_{13}} \\
& +8 \frac{-s_{24} s_{34}+m_{H}{ }^{2} s_{34}}{s_{134} s_{13} s_{234}}+8 \frac{s_{34}{ }^{2}+s_{24} s_{34}+m_{H}{ }^{2} s_{34}}{s_{234} s_{134} s_{14}} \\
& +8 \frac{s_{34}^{2}+m_{H}^{2} s_{34}+s_{14} s_{34}}{s_{134} s_{234} s_{24}}+8 \frac{-s_{14} s_{34}+m_{H}^{2} s_{34}}{s_{234} s_{134} s_{23}} \\
& +8 \frac{m_{H}^{2}}{s_{134}{ }^{2}}+8 \frac{m_{H}^{2}}{s_{234}{ }^{2}}-8 \frac{s_{34}+m_{H}^{2}+s_{24}-s_{13}}{s_{234} s_{14}}
\end{aligned}
$$




$$
\begin{aligned}
& +8 \frac{-s_{23}-s_{14}-s_{34}+m_{H}^{2}}{s_{24} s_{13}}+8 \frac{s_{24}+s_{14}}{s_{134} s_{23}}+8 \frac{-s_{23}+m_{H}^{2}}{s_{134} s_{13}} \\
& -8 \frac{m_{H}^{2}+s_{34}-s_{23}+s_{14}}{s_{134} s_{24}}+8 \frac{s_{24}+s_{14}}{s_{234} s_{13}} \\
& +8 \frac{m_{H}^{2}-s_{34}-s_{13}-s_{24}}{s_{14} s_{23}}+16 \frac{2 m_{H}^{2}+2 s_{34}}{s_{134} s_{234}} \\
& C_{H b \bar{b} g g}^{(0)}=8 \frac{2 s_{24}+4 m_{H}^{2}+s_{14}-s_{13}}{s_{234} s_{34}}-4 \frac{-s_{14}-2 s_{13}+4 m_{H}^{2}-4 s_{23}}{s_{24} s_{34}} \\
& -4 \frac{-2 s_{14}-2 s_{23}+2 m_{H}^{2}-s_{24}}{s_{13} s_{34}}-4 \frac{2 m_{H}^{2}-2 s_{13}-s_{14}-2 s_{24}}{s_{23} s_{34}} \\
& +16 \frac{-m_{H}^{2} s_{24}-4 m_{H}^{4}-2 s_{24} s_{14}-s_{14}^{2}-s_{24}^{2}-m_{H}^{2} s_{14}}{s_{134} s_{34} s_{234}} \\
& -4 \frac{-s_{24}{ }^{2}+2 m_{H}{ }^{2} s_{24}-2 m_{H}{ }^{4}-s_{14}{ }^{2}+2 m_{H}{ }^{2} s_{14}-2 s_{24} s_{14}}{s_{234} s_{34} s_{13}} \\
& -4 \frac{2 s_{24} s_{13}-2 m_{H}{ }^{4}-s_{24}{ }^{2}-s_{13}{ }^{2}+2 m_{H}{ }^{2} s_{13}-2 m_{H}{ }^{2} s_{24}}{s_{234} s_{34} s_{14}} \\
& -32 \frac{s_{24} s_{14} m_{H}^{2}}{s_{134} s_{34}{ }^{2} s_{234}}-4 \frac{-s_{24}-2 s_{23}+4 m_{H}{ }^{2}-4 s_{13}}{s_{14} s_{34}} \\
& -4 \frac{2 s_{14} s_{23}-s_{23}{ }^{2}+2 m_{H}{ }^{2} s_{23}-2 m_{H}{ }^{4}-s_{14}{ }^{2}-2 m_{H}{ }^{2} s_{14}}{s_{134} s_{34} s_{24}} \\
& -8 \frac{-s_{24}-4 m_{H}^{2}-2 s_{14}+s_{23}}{s_{134} s_{34}}+16 \frac{m_{H}^{2} s_{24}}{s_{234} s_{34}} \\
& -4 \frac{2 m_{H}^{2} s_{24}+2 m_{H}^{2} s_{13}-2 m_{H}^{4}-s_{24}^{2}-2 s_{24} s_{13}-s_{13}^{2}}{s_{14} s_{23} s_{34}} \\
& +16 \frac{s_{24}^{2} m_{H}^{2}}{s_{34}{ }^{2} s_{234}{ }^{2}}+16 \frac{m_{H}^{2} s_{14}}{s_{134} s_{34}}+16 \frac{s_{14}{ }^{2} m_{H}{ }^{2}}{s_{34}{ }^{2} s_{134}{ }^{2}} \\
& -4 \frac{-2 s_{14} s_{23}+2 m_{H}^{2} s_{23}-2 m_{H}^{4}-s_{23}^{2}+2 m_{H}^{2} s_{14}-s_{14}^{2}}{s_{24} s_{13} s_{34}} \\
& -4 \frac{-s_{24}{ }^{2}+2 m_{H}^{2} s_{24}-2 m_{H}^{4}-s_{14}^{2}+2 m_{H}^{2} s_{14}-2 s_{24} s_{14}}{s_{134} s_{34} s_{23}} \\
& C_{H b \bar{b} g g}^{(1)}=8 \frac{-s_{14}-2 s_{24}+s_{13}}{s_{234} s_{34}}-4 \frac{s_{24}+2 s_{23}+s_{14}}{s_{13} s_{34}}-4 \frac{s_{14}+s_{24}+2 s_{13}}{s_{23} s_{34}} \\
& +32 \frac{s_{24} s_{14} m_{H}^{2}}{s_{134} s_{34}{ }^{2} s_{234}}-4 \frac{2 s_{23}+4 s_{13}+s_{24}}{s_{14} s_{34}}-4 \frac{-3 s_{14} s_{23}+s_{23}{ }^{2}+s_{14}{ }^{2}}{s_{134} s_{34} s_{24}} \\
& -8 \frac{2 s_{14}+s_{24}-s_{23}}{s_{134} s_{34}}-16 \frac{m_{H}^{2} s_{24}}{s_{234}{ }^{2} s_{34}}-4 \frac{3 s_{24} s_{13}+s_{13}{ }^{2}+s_{24}{ }^{2}}{s_{14} s_{23} s_{34}} \\
& -16 \frac{s_{24}^{2} m_{H}^{2}}{s_{34}{ }^{2} s_{234}{ }^{2}}-4 \frac{4 s_{23}+s_{14}+2 s_{13}}{s_{24} s_{34}}-16 \frac{m_{H}^{2} s_{14}}{s_{134}{ }^{2} s_{34}} \\
& +16 \frac{m_{H}^{2} s_{14}+2 s_{24} s_{14}+m_{H}^{2} s_{24}+s_{14}^{2}+s_{24}^{2}}{s_{134} s_{34} s_{234}}-16 \frac{s_{14}{ }^{2} m_{H}^{2}}{s_{34}{ }^{2} s_{134}{ }^{2}} \\
& -4 \frac{s_{24}^{2}+s_{14}^{2}+3 s_{24} s_{14}}{s_{234} s_{34} s_{13}}-4 \frac{s_{24}^{2}+s_{14}^{2}+3 s_{24} s_{14}}{s_{134} s_{34} s_{23}} \\
& -4 \frac{s_{14}^{2}+3 s_{14} s_{23}+s_{23}^{2}}{s_{24} s_{13} s_{34}}-4 \frac{s_{13}^{2}+s_{24}^{2}-3 s_{24} s_{13}}{s_{234} s_{34} s_{14}}
\end{aligned}
$$




\section{References}

[1] P. Baikov, K. Chetyrkin and J.H. Kuhn, Scalar correlator at $O\left(\alpha_{s}^{4}\right)$, Higgs decay into b-quarks and bounds on the light quark masses, Phys. Rev. Lett. 96 (2006) 012003 [hep-ph/0511063] [INSPIRE].

[2] S. Gorishnii, A. Kataev and S. Larin, The width of Higgs boson decay into hadrons: three loop corrections of strong interactions, Sov. J. Nucl. Phys. 40 (1984) 329 [inSPIRE].

[3] S. Gorishnii, A. Kataev, S. Larin and L. Surguladze, Corrected three loop QCD correction to the correlator of the quark scalar currents and $\Gamma_{\text {tot }}\left(H^{0} \rightarrow\right.$ hadrons $)$, Mod. Phys. Lett. A 5 (1990) 2703 [INSPIRE].

[4] S. Gorishnii, A. Kataev, S. Larin and L. Surguladze, Scheme dependence of the next to next-to-leading $Q C D$ corrections to $\Gamma_{\text {tot }}\left(H^{0} \rightarrow\right.$ hadrons) and the spurious $Q C D$ infrared fixed point, Phys. Rev. D 43 (1991) 1633 [InSPIRE].

[5] C. Becchi, S. Narison, E. de Rafael and F. Yndurain, Light quark masses in quantum chromodynamics and chiral symmetry breaking, Z. Phys. C 8 (1981) 335 [INSPIRE].

[6] N. Sakai, Perturbative QCD corrections to the hadronic decay width of the Higgs boson, Phys. Rev. D 22 (1980) 2220 [INSPIRE].

[7] T. Inami and T. Kubota, Renormalization group estimate of the hadronic decay width of the Higgs boson, Nucl. Phys. B 179 (1981) 171 [inSPIRE].

[8] K. Chetyrkin, Correlator of the quark scalar currents and $\Gamma_{\text {tot }}\left(H \rightarrow\right.$ hadrons) at $O\left(\alpha_{S}^{3}\right)$ in pQCD, Phys. Lett. B 390 (1997) 309 [hep-ph/9608318] [inSPIRE].

[9] K. Chetyrkin and M. Steinhauser, Complete QCD corrections of order $O\left(\alpha_{S}^{3}\right)$ to the hadronic Higgs decay, Phys. Lett. B 408 (1997) 320 [hep-ph/9706462] [INSPIRE].

[10] J.M. Butterworth, A.R. Davison, M. Rubin and G.P. Salam, Jet substructure as a new Higgs search channel at the LHC, Phys. Rev. Lett. 100 (2008) 242001 [arXiv:0802.2470] [INSPIRE].

[11] T. Plehn, G.P. Salam and M. Spannowsky, Fat jets for a light Higgs, Phys. Rev. Lett. 104 (2010) 111801 [arXiv:0910.5472] [INSPIRE].

[12] ATLAS collaboration, ATLAS sensitivity to the standard model Higgs in the $H W$ and $H Z$ channels at High transverse momenta, PHYS-PUB-2009-088 (2009).

[13] D.E. Soper and M. Spannowsky, Combining subjet algorithms to enhance ZH detection at the LHC, JHEP 08 (2010) 029 [arXiv: 1005.0417] [INSPIRE].

[14] A. Bredenstein, A. Denner, S. Dittmaier and S. Pozzorini, NLO QCD corrections to top anti-top bottom anti-bottom production at the LHC: 2. full hadronic results, JHEP 03 (2010) 021 [arXiv: 1001.4006] [INSPIRE].

[15] A. Denner, S. Dittmaier, S. Kallweit and S. Pozzorini, NLO QCD corrections to WWbb production at hadron colliders, Phys. Rev. Lett. 106 (2011) 052001 [arXiv:1012.3975] [INSPIRE].

[16] T. Melia, K. Melnikov, R. Rontsch and G. Zanderighi, Next-to-leading order QCD predictions for $W^{+} W^{+} j j$ production at the LHC, JHEP 12 (2010) 053 [arXiv:1007.5313] [INSPIRE]. 
[17] R. Ellis, K. Melnikov and G. Zanderighi, $W+3$ jet production at the Tevatron, Phys. Rev. D 80 (2009) 094002 [arXiv:0906.1445] [INSPIRE].

[18] C. Berger et al., Precise predictions for $W+4$ jet production at the large hadron collider, Phys. Rev. Lett. 106 (2011) 092001 [arXiv:1009.2338] [INSPIRE].

[19] C. Berger et al., Next-to-leading order QCD predictions for $Z, \gamma^{*}+3$-jet distributions at the Tevatron, Phys. Rev. D 82 (2010) 074002 [arXiv:1004.1659] [INSPIRE].

[20] C. Berger et al., Next-to-leading order QCD Predictions for $W+3$-jet distributions at hadron colliders, Phys. Rev. D 80 (2009) 074036 [arXiv:0907.1984] [INSPIRE].

[21] H. Ita et al., Precise predictions for $Z+4$ jets at hadron colliders, Phys. Rev. D 85 (2012) 031501 [arXiv:1108.2229] [INSPIRE].

[22] G. Bevilacqua, M. Czakon, C. Papadopoulos, R. Pittau and M. Worek, Assault on the NLO Wishlist: $p p \rightarrow t \bar{t} b \bar{b}, J H E P 09$ (2009) 109 [arXiv:0907.4723] [InSPIRE].

[23] G. Bevilacqua, M. Czakon, C. Papadopoulos and M. Worek, Dominant QCD backgrounds in Higgs boson analyses at the LHC: A study of $p p \rightarrow t \bar{t}+2$ jets at next-to-leading order, Phys. Rev. Lett. 104 (2010) 162002 [arXiv:1002.4009] [INSPIRE].

[24] G. Bevilacqua, M. Czakon, A. van Hameren, C.G. Papadopoulos and M. Worek, Complete off-shell effects in top quark pair hadroproduction with leptonic decay at next-to-leading order, JHEP 02 (2011) 083 [arXiv: 1012.4230] [INSPIRE].

[25] N. Greiner, A. Guffanti, J.-P. Guillet, T. Reiter and J. Reuter, NLO QCD corrections to 4 b-quark production, PoS(DIS 2010) 156 [arXiv:1006.5339] [INSPIRE].

[26] R. Frederix et al., $W$ and $Z / \gamma *$ boson production in association with a bottom-antibottom pair, JHEP 09 (2011) 061 [arXiv:1106.6019] [INSPIRE].

[27] C. Berger et al., An automated implementation of on-shell methods for one-loop amplitudes, Phys. Rev. D 78 (2008) 036003 [arXiv:0803.4180] [INSPIRE].

[28] R. Ellis, W.T. Giele, Z. Kunszt and K. Melnikov, Masses, fermions and generalized D-dimensional unitarity, Nucl. Phys. B 822 (2009) 270 [arXiv:0806.3467] [INSPIRE].

[29] A. van Hameren, C. Papadopoulos and R. Pittau, Automated one-loop calculations: a proof of concept, JHEP 09 (2009) 106 [arXiv:0903.4665] [INSPIRE].

[30] V. Hirschi et al., Automation of one-loop QCD corrections, JHEP 05 (2011) 044 [arXiv: 1103.0621] [INSPIRE].

[31] R. Frederix, S. Frixione, F. Maltoni and T. Stelzer, Automation of next-to-leading order computations in QCD: the FKS subtraction, JHEP 10 (2009) 003 [arXiv:0908.4272] [INSPIRE].

[32] R. Frederix, T. Gehrmann and N. Greiner, Automation of the dipole subtraction method in MadGraph/MadEvent, JHEP 09 (2008) 122 [arXiv:0808.2128] [INSPIRE].

[33] T. Gleisberg and F. Krauss, Automating dipole subtraction for QCD NLO calculations, Eur. Phys. J. C 53 (2008) 501 [arXiv:0709.2881] [InSPIRE].

[34] T. Binoth, J.-P. Guillet, G. Heinrich, E. Pilon and T. Reiter, Golem95: a numerical program to calculate one-loop tensor integrals with up to six external legs,

Comput. Phys. Commun. 180 (2009) 2317 [arXiv:0810.0992] [INSPIRE]. 
[35] P. Mastrolia, G. Ossola, T. Reiter and F. Tramontano, Scattering AMplitudes from unitarity-based reduction algorithm at the integrand-level, JHEP 08 (2010) 080 [arXiv: 1006.0710] [INSPIRE].

[36] D.A. Kosower, Antenna factorization of gauge theory amplitudes, Phys. Rev. D 57 (1998) 5410 [hep-ph/9710213] [INSPIRE].

[37] A. Gehrmann-De Ridder, T. Gehrmann and G. Heinrich, Four particle phase space integrals in massless QCD, Nucl. Phys. B 682 (2004) 265 [hep-ph/0311276] [INSPIRE].

[38] A. Gehrmann-De Ridder, T. Gehrmann and E. Glover, Antenna subtraction at NNLO, JHEP 09 (2005) 056 [hep-ph/0505111] [INSPIRE].

[39] A. Daleo, T. Gehrmann and D. Maître, Antenna subtraction with hadronic initial states, JHEP 04 (2007) 016 [hep-ph/0612257] [INSPIRE].

[40] A. Gehrmann-De Ridder and M. Ritzmann, NLO antenna subtraction with massive fermions, JHEP 07 (2009) 041 [arXiv: 0904.3297] [INSPIRE].

[41] A. Daleo, A. Gehrmann-De Ridder, T. Gehrmann and G. Luisoni, Antenna subtraction at NNLO with hadronic initial states: initial-final configurations, JHEP 01 (2010) 118 [arXiv: 0912.0374] [INSPIRE].

[42] E. Nigel Glover and J. Pires, Antenna subtraction for gluon scattering at NNLO, JHEP 06 (2010) 096 [arXiv: 1003.2824] [INSPIRE].

[43] R. Boughezal, A. Gehrmann-De Ridder and M. Ritzmann, Antenna subtraction at NNLO with hadronic initial states: double real radiation for initial-initial configurations with two quark flavours, JHEP 02 (2011) 098 [arXiv: 1011.6631] [INSPIRE].

[44] G. Abelof and A. Gehrmann-De Ridder, Antenna subtraction for the production of heavy particles at hadron colliders, JHEP 04 (2011) 063 [arXiv:1102.2443] [INSPIRE].

[45] T. Gehrmann and P.F. Monni, Antenna subtraction at NNLO with hadronic initial states: real-virtual initial-initial configurations, JHEP 12 (2011) 049 [arXiv:1107.4037] [INSPIRE].

[46] S. Weinzierl, Subtraction terms at NNLO, JHEP 03 (2003) 062 [hep-ph/0302180] [INSPIRE].

[47] S. Frixione and M. Grazzini, Subtraction at NNLO, JHEP 06 (2005) 010 [hep-ph/0411399] [INSPIRE].

[48] G. Somogyi, Z. Trócsányi and V. Del Duca, Matching of singly- and doubly-unresolved limits of tree-level QCD squared matrix elements, JHEP 06 (2005) 024 [hep-ph/0502226] [INSPIRE].

[49] G. Somogyi, Z. Trócsányi and V. Del Duca, A subtraction scheme for computing QCD jet cross sections at NNLO: regularization of doubly-real emissions, JHEP 01 (2007) 070 [hep-ph/0609042] [INSPIRE].

[50] G. Somogyi and Z. Trócsányi, A subtraction scheme for computing QCD jet cross sections at NNLO: regularization of real-virtual emission, JHEP 01 (2007) 052 [hep-ph/0609043] [INSPIRE].

[51] P. Bolzoni, S.-O. Moch, G. Somogyi and Z. Trócsányi, Analytic integration of real-virtual counterterms in NNLO jet cross sections. II., JHEP 08 (2009) 079 [arXiv:0905.4390] [INSPIRE]. 
[52] P. Bolzoni, G. Somogyi and Z. Trócsányi, A subtraction scheme for computing QCD jet cross sections at NNLO: integrating the iterated singly-unresolved subtraction terms, JHEP 01 (2011) 059 [arXiv: 1011.1909] [INSPIRE].

[53] G. Somogyi and Z. Trócsányi, A subtraction scheme for computing QCD jet cross sections at NNLO: integrating the subtraction terms. I., JHEP 08 (2008) 042 [arXiv:0807.0509] [INSPIRE].

[54] U. Aglietti, V. Del Duca, C. Duhr, G. Somogyi and Z. Trócsányi, Analytic integration of real-virtual counterterms in NNLO jet cross sections. I., JHEP 09 (2008) 107 [arXiv: 0807.0514] [INSPIRE].

[55] M. Czakon, A novel subtraction scheme for double-real radiation at NNLO, Phys. Lett. B 693 (2010) 259 [arXiv: 1005.0274] [INSPIRE].

[56] M. Czakon, Double-real radiation in hadronic top quark pair production as a proof of a certain concept, Nucl. Phys. B 849 (2011) 250 [arXiv:1101.0642] [INSPIRE].

[57] C. Anastasiou, K. Melnikov and F. Petriello, A new method for real radiation at NNLO, Phys. Rev. D 69 (2004) 076010 [hep-ph/0311311] [InSPIRE].

[58] S. Catani and M. Grazzini, An NNLO subtraction formalism in hadron collisions and its application to Higgs boson production at the LHC, Phys. Rev. Lett. 98 (2007) 222002 [hep-ph/0703012] [INSPIRE].

[59] T. Binoth and G. Heinrich, An automatized algorithm to compute infrared divergent multiloop integrals, Nucl. Phys. B 585 (2000) 741 [hep-ph/0004013] [INSPIRE].

[60] T. Binoth and G. Heinrich, Numerical evaluation of phase space integrals by sector decomposition, Nucl. Phys. B 693 (2004) 134 [hep-ph/0402265] [INSPIRE].

[61] G. Ferrera, M. Grazzini and F. Tramontano, Associated WH production at hadron colliders: a fully exclusive QCD calculation at NNLO, Phys. Rev. Lett. 107 (2011) 152003 [arXiv:1107.1164] [INSPIRE].

[62] S. Catani, L. Cieri, G. Ferrera, D. de Florian and M. Grazzini, Vector boson production at hadron colliders: a fully exclusive QCD calculation at NNLO, Phys. Rev. Lett. 103 (2009) 082001 [arXiv:0903.2120] [INSPIRE].

[63] K. Melnikov and F. Petriello, Electroweak gauge boson production at hadron colliders through $O\left(\alpha_{s}^{2}\right)$, Phys. Rev. D 74 (2006) 114017 [hep-ph/0609070] [INSPIRE].

[64] C. Anastasiou, L.J. Dixon, K. Melnikov and F. Petriello, Dilepton rapidity distribution in the Drell-Yan process at NNLO in QCD, Phys. Rev. Lett. 91 (2003) 182002 [hep-ph/0306192] [INSPIRE].

[65] C. Anastasiou, L.J. Dixon, K. Melnikov and F. Petriello, High precision QCD at hadron colliders: electroweak gauge boson rapidity distributions at NNLO, Phys. Rev. D 69 (2004) 094008 [hep-ph/0312266] [INSPIRE].

[66] C. Anastasiou, L.J. Dixon and K. Melnikov, NLO Higgs boson rapidity distributions at hadron colliders, Nucl. Phys. Proc. Suppl. 116 (2003) 193 [hep-ph/0211141] [InSPIRE].

[67] C. Anastasiou, K. Melnikov and F. Petriello, Higgs boson production at hadron colliders: Differential cross sections through next-to-next-to-leading order, Phys. Rev. Lett. 93 (2004) 262002 [hep-ph/0409088] [INSPIRE]. 
[68] C. Anastasiou, K. Melnikov and F. Petriello, Fully differential Higgs boson production and the di-photon signal through next-to-next-to-leading order, Nucl. Phys. B 724 (2005) 197 [hep-ph/0501130] [INSPIRE].

[69] M. Grazzini, NNLO predictions for the Higgs boson signal in the $H \rightarrow W W \rightarrow l \nu l \nu$ and $H \rightarrow Z Z \rightarrow 4 l$ decay channels, JHEP 02 (2008) 043 [arXiv:0801.3232] [INSPIRE].

[70] C. Anastasiou, K. Melnikov and F. Petriello, Real radiation at NNLO: $e^{+} e^{-} \rightarrow 2$ jets through $O\left(\alpha_{s}^{2}\right)$, Phys. Rev. Lett. 93 (2004) 032002 [hep-ph/0402280] [INSPIRE].

[71] A. Gehrmann-De Ridder, T. Gehrmann and E. Glover, Infrared structure of $e^{+} e^{-} \rightarrow 2$ jets at NNLO, Nucl. Phys. B 691 (2004) 195 [hep-ph/0403057] [INSPIRE].

[72] A. Gehrmann-De Ridder, T. Gehrmann, E. Glover and G. Heinrich, NNLO corrections to event shapes in $e^{+} e^{-}$annihilation, JHEP 12 (2007) 094 [arXiv:0711.4711] [INSPIRE].

[73] A. Gehrmann-De Ridder, T. Gehrmann, E. Glover and G. Heinrich, Infrared structure of $e^{+} e^{-} \rightarrow 3$ jets at NNLO, JHEP 11 (2007) 058 [arXiv:0710.0346] [INSPIRE].

[74] C. Anastasiou, F. Herzog and A. Lazopoulos, On the factorization of overlapping singularities at NNLO, JHEP 03 (2011) 038 [arXiv:1011.4867] [INSPIRE].

[75] R.V. Harlander and W.B. Kilgore, Higgs boson production in bottom quark fusion at next-to-next-to leading order, Phys. Rev. D 68 (2003) 013001 [hep-ph/0304035] [INSPIRE].

[76] P. Nogueira, Automatic Feynman graph generation, J. Comput. Phys. 105 (1993) 279 [INSPIRE].

[77] J. Vermaseren, New features of FORM, math-ph/0010025 [INSPIRE].

[78] MAPLE, http://www.maplesoft.com.

[79] K. Chetyrkin and F. Tkachov, Integration by parts: the algorithm to calculate $\beta$-functions in 4 loops, Nucl. Phys. B 192 (1981) 159 [INSPIRE].

[80] F. Tkachov, a theorem on analytical calculability of four loop renormalization group functions, Phys. Lett. B 100 (1981) 65 [INSPIRE].

[81] S. Laporta, High precision calculation of multiloop Feynman integrals by difference equations, Int. J. Mod. Phys. A 15 (2000) 5087 [hep-ph/0102033] [inSPIRE].

[82] C. Anastasiou and A. Lazopoulos, Automatic integral reduction for higher order perturbative calculations, JHEP 07 (2004) 046 [hep-ph/0404258] [INSPIRE].

[83] S. Biswas and K. Melnikov, Second order QCD corrections to inclusive semileptonic $b \rightarrow X_{c} l \bar{\nu}_{l}$ decays with massless and massive lepton, JHEP 02 (2010) 089 [arXiv:0911.4142] [INSPIRE].

[84] JADE collaboration, W. Bartel et al., Experimental studies on multi-jet production in $e^{+} e^{-}$ annihilation at PETRA energies, Z. Phys. C 33 (1986) 23 [INSPIRE]. 